\title{
Insilico Analysis Reveal Three novel nsSNPs May effect on $G M 2 A$ protein Leading to AB variant of GM2 gangliosidosis
}

Tebyan A. Abdelhameed ${ }^{1}$, Mujahed I. Mustafa, ${ }^{1,2}$, Dina N. Abdelrahman ${ }^{1,3}$, Fatima A. Abdelrhman ${ }^{1}$, Mohamed A. Hassan ${ }^{1}$.

1. Department of biotechnology, Africa City of Technology-Khartoum, Sudan.

2. Department of Biochemistry, University of Bahri-Khartoum, Sudan.

3. Central laboratory, Ministry of Higher education and scientific research- Khartoum, Sudan.

Corresponding author: Tebyan A. Abdelhameed: tebyanameer2511@gmail.com.

\begin{abstract}
Background: $\mathrm{AB}$ variant of GM2 gangliosidosis caused as a result of mutations in GM2 activator gene $(G M 2 A)$ which is regarded as neurodegenerative disorder. This study aimed to predict the possible damaging SNPs of this gene and their impact on the protein using different bioinformatics tools. Methods: SNPs retrieved from the NCBI database were analyzed using several bioinformatics tools. The different tools collectively predicted the effect of single nucleotide substitution on both structure and function of GM2 activator. Results: Three novel mutations were found to be highly damaging to the structure and function of the $G M 2 A$ gene. Conclusion: Four SNPs were found to be highly damaging SNPs that affect function, structure and stability of GM2A protein, which they are: C99Y, $\mathrm{C} 112 \mathrm{~F}, \mathrm{C} 138 \mathrm{~S}$ and C138R, three of them are novel SNPs (C99Y, C112F and C138S). Also 46 SNPs were predicted to affect miRNAs binding sites on 3'UTR leading to abnormal expression of the resulting protein. These SNPs should be considered as important candidates in causing $\mathrm{AB}$ variant of GM2 gangliosidosis and may help in diagnosis and genetic screening of the disease.
\end{abstract}

Keywords: GM2 gangliosidosis- $A B$ variant, GM2A, neurodegenerative disorder, bioinformatics, single nucleotide polymorphisms (SNPS), Computational, insilico. 


\section{INTRODUCTION:}

GM2-gangliosidosis is a group of neurological disorders resulting from genetically defective catabolism, and consequent abnormal accumulation, of GM2-ganglioside. Three major types are distinguished: the $\mathrm{B}$ variant (Tay-Sachs disease), the $\mathrm{O}$ variant (Sandhoff disease), and the $\mathrm{AB}$ variant, caused by genetic abnormalities in the genes coding for the beta-hexosaminidase alpha- or beta-subunit, or the GM2-activator protein, respectively. In this study we will focus only in the AB variant of GM2 gangliosidosis.(1, 2) Variant AB of infantile GM2 gangliosidosis is a fatal disease leading invariably to death within the first few years of life, due to the excessive storage of the glycolipids GM2 and GA2 which occurs in the nervous tissue of the patient. $(3,4)$ The variant $A B$ is characterized by a normal or close to the normal range of this enzyme. Thus, variant $\mathrm{AB}$ is an example of a fatal lipid storage disease that is caused not by a defect of a degrading enzyme but rather by a defective factor necessary for the interaction of lipid substrates and the water-soluble hydrolase. (3, 5-7)

The most reported gene for this disorder is $G M 2 A(8,9)$ which acts as a cofactor for the enzyme. $(10,11)$ located in chromosome 5q31.3-q33.1 (12-14) The GM2 activator deficiency is caused by mutations in the GM2A gene encoding the GM2 activator protein.(15) Different mutations have been reported related to $G M 2 A$, (16-22) but interestingly, some study shows that, mutation in $G M 2 A$ Leads to a Progressive Chorea-dementia Syndrome.(23)

ELISA system can be used as diagnosis tool as well as therapeutic evaluation of GM2 gangliosidoses using anti-GM2 ganglioside antibodies(24) Can also be diagnosed by demonstrating accumulation of GM2 in the CSF of patients with normal hexosaminidase activity.(25) Biochemical and molecular analysis can also be additional diagnosis approaches.(10, 26-28) Some study revealed that, not all GM2-gangliosidosis pateints can have mutations in the protein coding region of the GM2 activator gene.(29)

The usage of in silico studies has strong impact on the identifcation of candidate SNPs since they are easy and less costly and can facilitate future genetic studies.(30) The main objective of this study was to identify the pathogenic SNPs in coding region and 3'UTR in GM2A gene using in silico analysis sofwares and to determine the effect of these SNPs on the structural, functional levels, and regulation of their respective proteins.

\section{MATERIALS AND METHODS:}

\subsection{Data mining:}

The data on human GM2A gene was collected from National Center for Biological Information (NCBI) web site (31). The SNP information (protein accession number and SNP ID) of the MEFV gene was retrieved from the NCBI dbSNP (http://www.ncbi.nlm.nih.gov/snp/) and the protein sequence was collected from Swiss Prot databases (http://expasy.org/ )(32). 


\subsection{SIFT:}

SIFT is a sequence homology-based tool (33) that sorts intolerant from tolerant amino acid substitutions and predicts whether an aminoacid substitution in a protein will have a phenotypic Effect. Considers the position at which the change occurred and the type of amino acid change. Given a protein sequence, SIFT chooses related proteins and obtains an alignment of these proteins with the query. Based on the amino acids appearing at each position in the alignment, SIFT calculates the probability that an amino acid at a position is tolerated conditional on the most frequent amino acid being tolerated. If this normalized value is less than a cutoff, the substitution is predicted to be deleterious. SIFT scores $<0.05$ are predicted by the algorithm to be intolerant or deleterious amino acid substitutions, whereas scores $>0.05$ are considered tolerant. It is available at (http://sift.bii.a-star.edu.sg/ ).

\subsection{Polyphen-2:}

It is a software tool (34) to predict possible impact of an amino acid substitution on both structure and function of a human protein by analysis of multiple sequence alignment and protein 3D structure, in addition it calculates position-specific independent count scores (PSIC) for each of two variants, and then calculates the PSIC scores difference between two variants. The higher a PSIC score difference, the higher the functional impact a particular amino acid substitution is likely to have. Prediction outcomes could be classified as probably damaging, possibly damaging or benign according to the value of PSIC as it ranges from $\left(0 \_1\right)$; values closer to zero considered benign while values closer to 1 considered probably damaging and also it can be indicated by a vertical black marker inside a color gradient bar, where green is benign and red is damaging. nsSNPs that predicted to be intolerant by Sift has been submitted to Polyphen as protein sequence in FASTA format that obtained from UniproktB /Expasy after submitting the relevant ensemble protein (ESNP) there, and then we entered position of mutation, native amino acid and the new substituent for both structural and functional predictions. PolyPhen version 2.2.2 is available at (http://genetics.bwh.harvard.edu/pph2/index.shtml ).

\subsection{Provean:}

Provean is a software tool (35) which predicts whether an amino acid substitution or indel has an impact on the biological function of a protein. it is useful for filtering sequence variants to identify nonsynonymous or indel variants that are predicted to be functionally important. It is available at (https://rostlab.org/services/snap2web/ ).

\subsection{SNAP2:}

Functional effects of mutations are predicted with SNAP2 (36). SNAP2 is a trained classifier that is based on a machine learning device called "neural network". It distinguishes between effect and neutral variants/non-synonymous SNPs by taking a variety of sequence and variant features into account. The most important input signal for the prediction is the evolutionary information taken from an automatically generated multiple sequence alignment. Also structural features such as predicted secondary structure and solvent accessibility are considered. If available also annotation (i.e. known functional residues, pattern, regions) of the sequence or close homologs are pulled in. In a cross-validation over 100,000 
experimentally annotated variants, SNAP2 reached sustained two-state accuracy (effect/neutral) of $82 \%$ (at an AUC of 0.9 ). In our hands this constitutes an important and significant improvement over other methods. It is available at (https://rostlab.org/services/snap2web/ ).

\subsection{PHD-SNP:}

An online Support Vector Machine (SVM) based classifier, is optimized to predict if a given single point protein mutation can be classified as disease-related or as a neutral polymorphism, it is available at: (http://http://snps.biofold.org/phd-snp/phdsnp.html).

\subsection{SNP\& Go:}

SNPs\&GO is an accurate method that, starting from a protein sequence, can predict whether a variation is disease related or not by exploiting the corresponding protein functional annotation. SNPs\&GO collects in unique framework information derived from protein sequence, evolutionary information, and function as encoded in the Gene Ontology terms, and outperforms other available predictive methods. (37) It is available at (http://snps.biofold.org/snps-and-go/snps-and-go.html )

\subsection{P-Mut:}

PMUT a web-based tool (38) for the annotation of pathological variants on proteins, allows the fast and accurate prediction (approximately 80\% success rate in humans) of the pathological character of single point amino acidic mutations based on the use of neural networks. It is available at (http://mmb.irbbarcelona.org/PMut ).

\subsection{I-Mutant 3.0:}

I-Mutant 3.0 Is a neural network based tool (39) for the routine analysis of protein stability and alterations by taking into account the single-site mutations. The FASTA sequence of protein retrieved from UniProt is used as an input to predict the mutational effect on protein stability. It is available at (http://gpcr2.biocomp.unibo.it/cgi/predictors/I-Mutant3.0/IMutant3.0.cgi ).

\subsection{Project Hope:}

Online software is available at: (http://www.cmbi.ru.nl/hope/method/). It is a web service where the user can submit a sequence and mutation. The software collects structural information from a series of sources, including calculations on the 3D protein structure, sequence annotations in UniProt and prediction from other software. It combines this information to give analysis for the effect of a certain mutation on the protein structure. HOPE will show the effect of that mutation in such a way that even those without a bioinformatics background can understand it. It allows the user to submit a protein sequence (can be FASTA or not) or an accession code of the protein of interest. In the next step, the user can indicate the mutated residue with a simple mouse click. In the final step, the user can simply click on one of the other 19 amino acid types that will become the mutant residue, and then full report well is generated (40). 


\subsection{PolymiRTS:}

PolymiRTS is a software used to predict 3UTR (un-translated region) polymorphism in microRNAs and their target sites available at (http://compbio.uthsc.edu/miRSNP/ ). It is a database of naturally occurring DNA variations in mocriRNAs (miRNA) seed region and miRNA target sites. MicroRNAs pair to the transcript of protein coding genes and cause translational repression or mRNA destabilization. SNPs in microRNA and their target sites may affect miRNA-mRNA interaction, causing an effect on miRNA-mediated gene repression, PolymiRTS database was created by scanning 3UTRs of mRNAs in human and mouse for SNPs in miRNA target sites. Then, the effect of polymorphism on gene expression and phenotypes are identified and then linked in the database. The PolymiRTS data base also includes polymorphism in target sites that have been supported by a variety of experimental methods and polymorphism in miRNA seed regions. (42)

\subsection{UCSF Chimera (University of California at San Francisco):}

UCSF Chimera (https://www.cgl.ucsf.edu/chimera/ ) is a highly extensible program for interactive visualization and analysis of molecular structures and related data, including density maps, supramolecular assemblies, sequence alignments, docking results, trajectories, and conformational ensembles. High-quality images and animations can be generated. Chimera includes complete documentation and several tutorials. Chimera is developed by the Resource for Biocomputing, Visualization, and Informatics (RBVI), supported by the National Institutes of Health (P41-GM103311).(41)

\subsection{Raptor X:}

RaptorX (http://raptorx.uchicago.edu/): It is a web server predicting structure property of a protein sequence without using any templates. It outperforms other servers, especially for proteins without close homologs in PDB or with very sparse sequence profile. The server predicts tertiary structure (43)

\subsection{GeneMANIA:}

It is gene interaction software that finds other genes which is related to a set of input genes using a very large set of functional association data. Association data include protein and genetic interactions, pathways, co-expression, co-localization and protein domain similarity. GeneMANIA also used to find new members of a pathway or complex, find additional genes you may have missed in your screen or find new genes with a specific function, such as protein kinases. available at (https://genemania.org/) (44).

\section{RESUTS:}

\subsection{Retrieval of SNPs from the Database:}

All SNPs data related to GM2A gene was gathered from dbSNP in National Center of Biotechnology Information (NCBI) database. This gene containing a total of 247 SNPs in coding region, of which 159 were missense, 74 synonymous, 5 nonsense, 8 frame shift, and 676 in 3'untranslated region (3' UTR). 


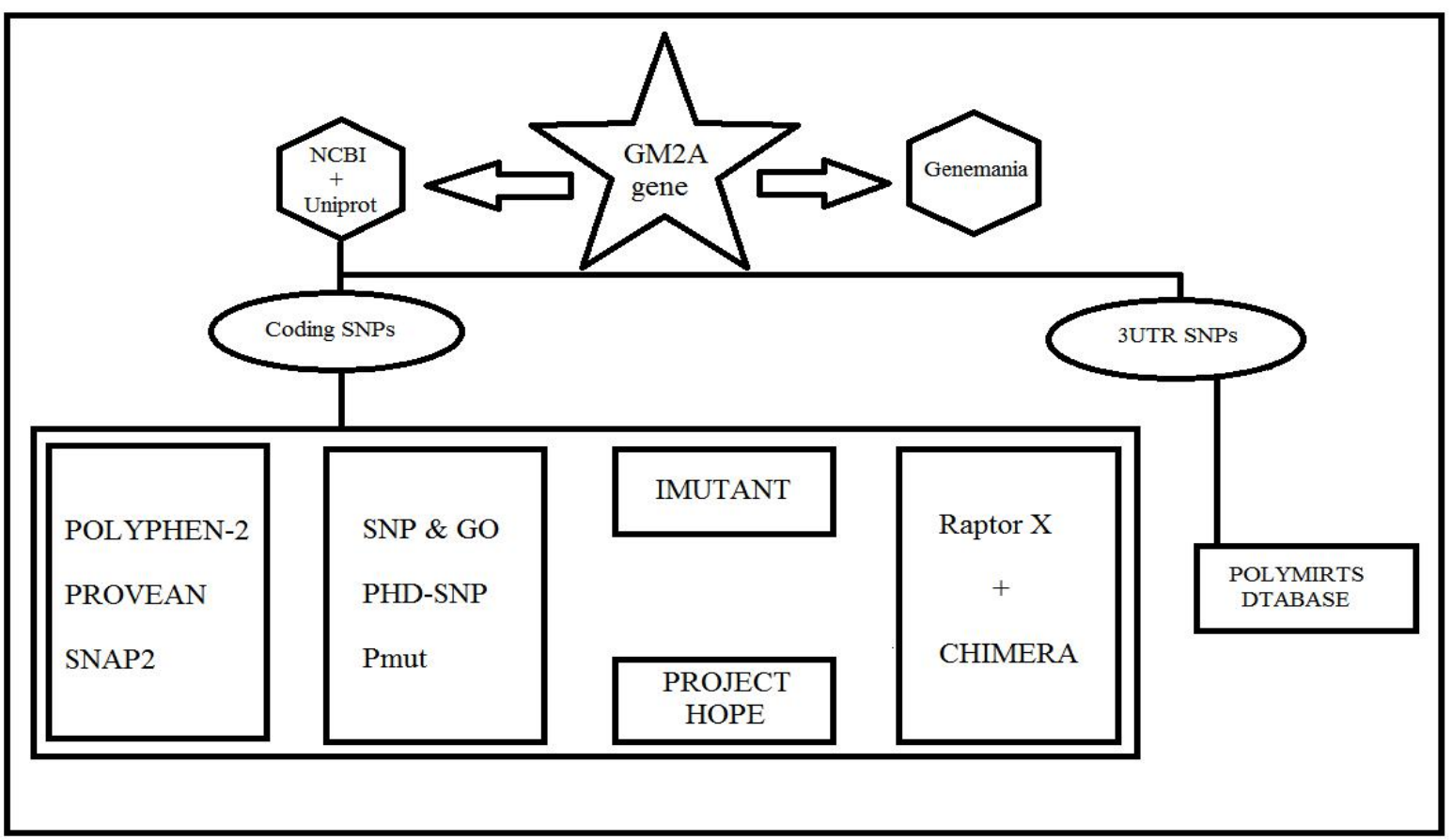

Figure (1): Flowchart of the software used in the analysis processes.

\subsection{The functional effect of Deleterious and damaging nsSNPs of $G M 2 A$ by SIFT, Provean PolyPhen-2, and SNAP2}

Table (1): Prediction of functional effect of Deleterious and damaging nsSNPs by SIFT, Polyphen-2, PROVEAN and SNAP2. 


\begin{tabular}{|l|l|l|l|l|l|l|}
\hline Variant & Polyphen2 Prediction & $\begin{array}{c}\text { Polyphen } \\
\text { 2 score }\end{array}$ & $\begin{array}{c}\text { PROVEAN } \\
\text { score }\end{array}$ & $\begin{array}{c}\text { PROVEAN } \\
\text { Prediction } \\
\text { (cutoff= -2.5) }\end{array}$ & $\begin{array}{c}\text { SNAP2 } \\
\text { Prediction }\end{array}$ & $\begin{array}{c}\text { SNAP2 } \\
\text { score }\end{array}$ \\
\hline C99Y & possibly damaging & 1 & -10.833 & Deleterious & effect & effect \\
\hline C112F & probably damaging & 1 & -10.8 & Deleterious & effect & 81 \\
\hline C138R & probably damaging & 1 & -12 & Deleterious & effect & 89 \\
\hline C138S & probably damaging & 1 & -10 & Deleterious & 84 \\
\hline
\end{tabular}

\subsection{Functional analysis of ADAMTS13 gene using Disease Related and pathological effect of nsSNPs by PhD-SNP, SNPs \& GO and PMut softwares:}

Table (2): Prediction of disease related nsSNPs through using: PhD-SNP, SNPs \& GO and PMut softwares.

\begin{tabular}{|c|c|c|c|c|c|c|c|c|c|}
\hline Mutation & $\begin{array}{l}\text { SNP } \\
\text { Prediction }\end{array}$ & $\begin{array}{l}\mathrm{R} \\
\mathrm{I}\end{array}$ & Probability & $\begin{array}{l}\mathrm{PhD} \\
\text { Prediction }\end{array}$ & $\begin{array}{l}\mathrm{R} \\
\mathrm{I}\end{array}$ & Probability & $\begin{array}{l}\text { Pmut } \\
\text { Position }\end{array}$ & $\begin{array}{l}\text { Mutatio } \\
\mathrm{n}\end{array}$ & Prediction \\
\hline C99Y & Disease & 7 & 0.831 & Disease & 9 & 0.949 & $\underline{99}$ & $\frac{\frac{\mathrm{C} \rightarrow \mathrm{Y}}{(\mathrm{Cys} \rightarrow}}{\underline{\text { Tyr })}}$ & $\begin{array}{l}0.82(90 \%) \\
\text { Disease }\end{array}$ \\
\hline $\mathrm{C} 112 \mathrm{~F}$ & Disease & 3 & 0.67 & Disease & 8 & 0.887 & $\underline{112}$ & $\begin{array}{l}\frac{\mathrm{C} \rightarrow \mathrm{F}}{(\mathrm{Cys} \rightarrow} \\
\underline{\underline{\text { Phe })}}\end{array}$ & $\begin{array}{l}0.82(90 \%) \\
\text { Disease }\end{array}$ \\
\hline C138R & Disease & 3 & 0.67 & Disease & 8 & 0.896 & $\underline{138}$ & $\begin{array}{l}\frac{\mathrm{C} \rightarrow \mathrm{R}}{(\mathrm{Cys} \rightarrow} \\
\underline{\operatorname{Arg})}\end{array}$ & $\begin{array}{l}0.82(90 \%) \\
\text { Disease }\end{array}$ \\
\hline $\mathrm{C} 138 \mathrm{~S}$ & Disease & 2 & 0.623 & Disease & 7 & 0.843 & 138 & $\begin{array}{l}\frac{\mathrm{C} \rightarrow \mathrm{S}}{(\mathrm{Cys} \rightarrow} \\
\underline{\text { Ser })}\end{array}$ & $\begin{array}{l}0.82(90 \%) \\
\text { Disease }\end{array}$ \\
\hline
\end{tabular}




\subsection{Prediction of Change in Stability due to Mutation Using I-Mutant 3.0 Server}

Table (3): Effect of nsSNPs on protein stability using I-Mutant

\begin{tabular}{|l|l|c|c|}
\hline & & & \\
dbSNP rs\# & Mutation & Prediction & DDG value (score) \\
\hline rs751417546 & & & \\
\hline & C99Y & Increase Protein Stability & -0.05 \\
\hline rs773743799 & C112F & Decrease Protein Stability & 0.03 \\
\hline rs137852797 & & & -0.24 \\
\hline & C138R & Decrease Protein Stability & \\
rs1174735558 & C138S & & -0.82 \\
\hline
\end{tabular}

3.5 Modeling of amino acid substitution effects on protein structure using Chimera and Project Hope Softwares. 


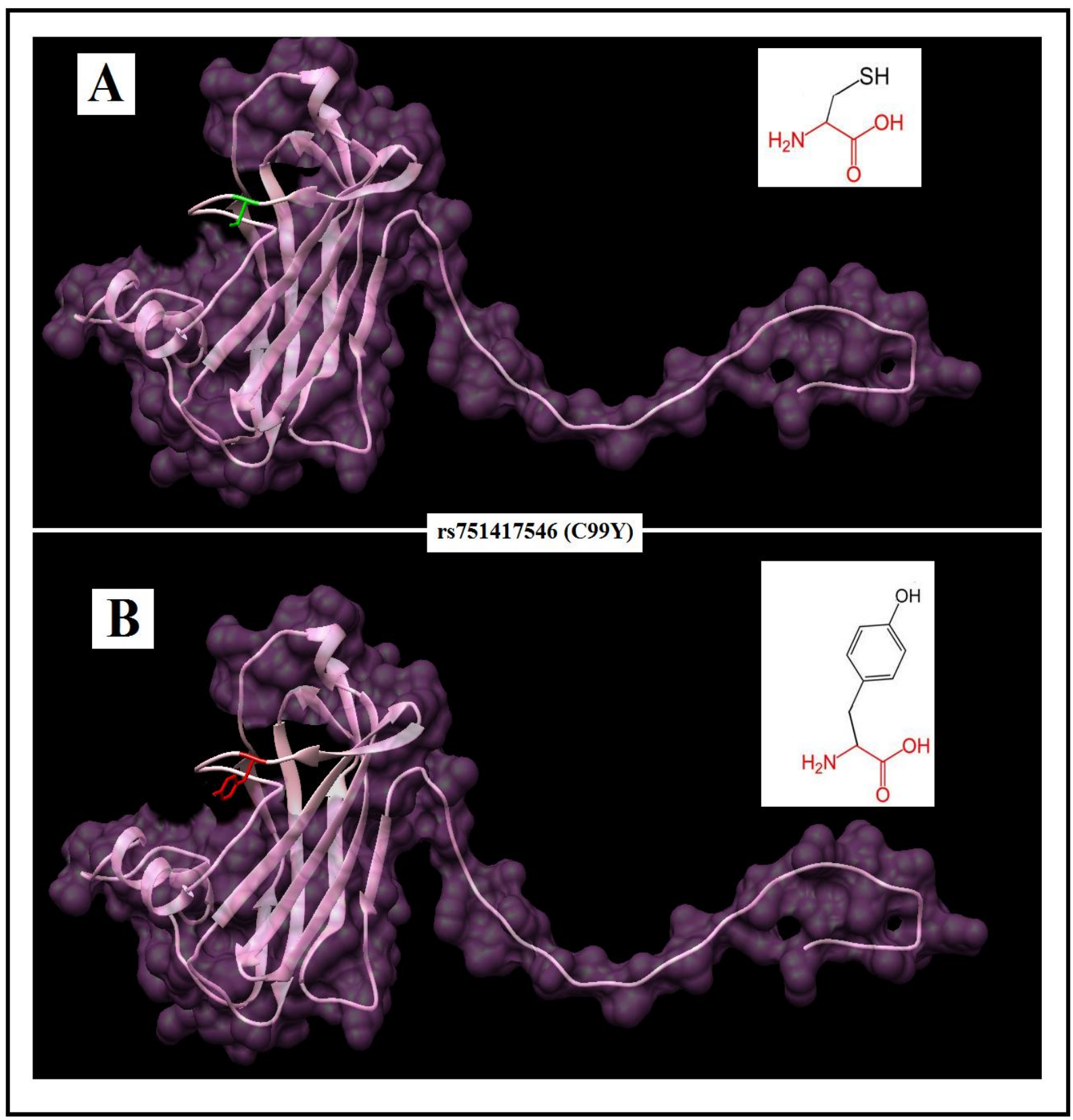

Figure (2): Effect of C99Y (rs751417546) SNP on protein structure in which Cysteine mutated into Tyrosine at position 99 using Project HOPE and Chimera.. 


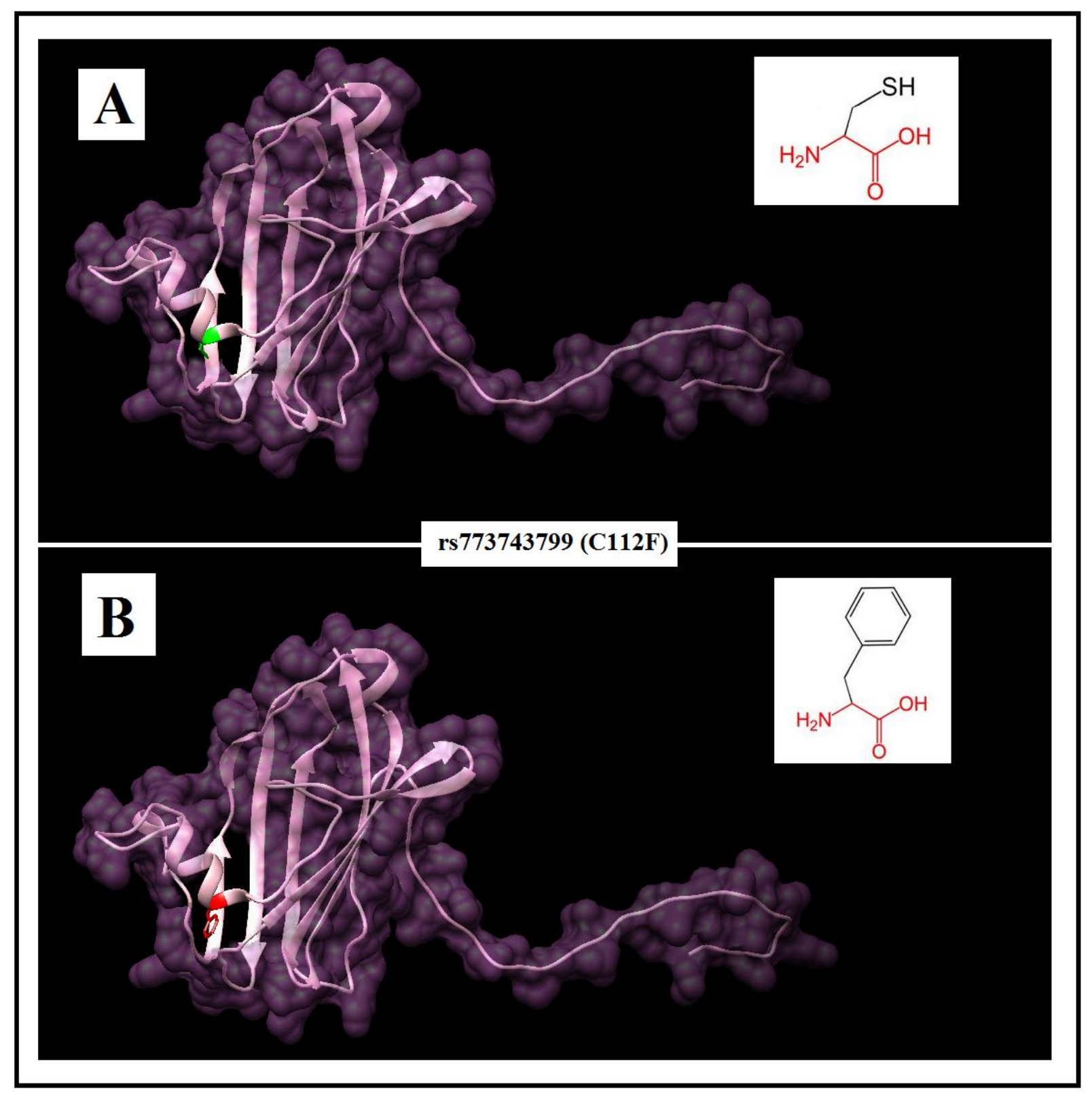

Figure (3): Effect of C112F (rs773743799) SNP on protein structure in which Cysteine mutated into Phenylalanine at position 112 using project HOPE and Chimera. 


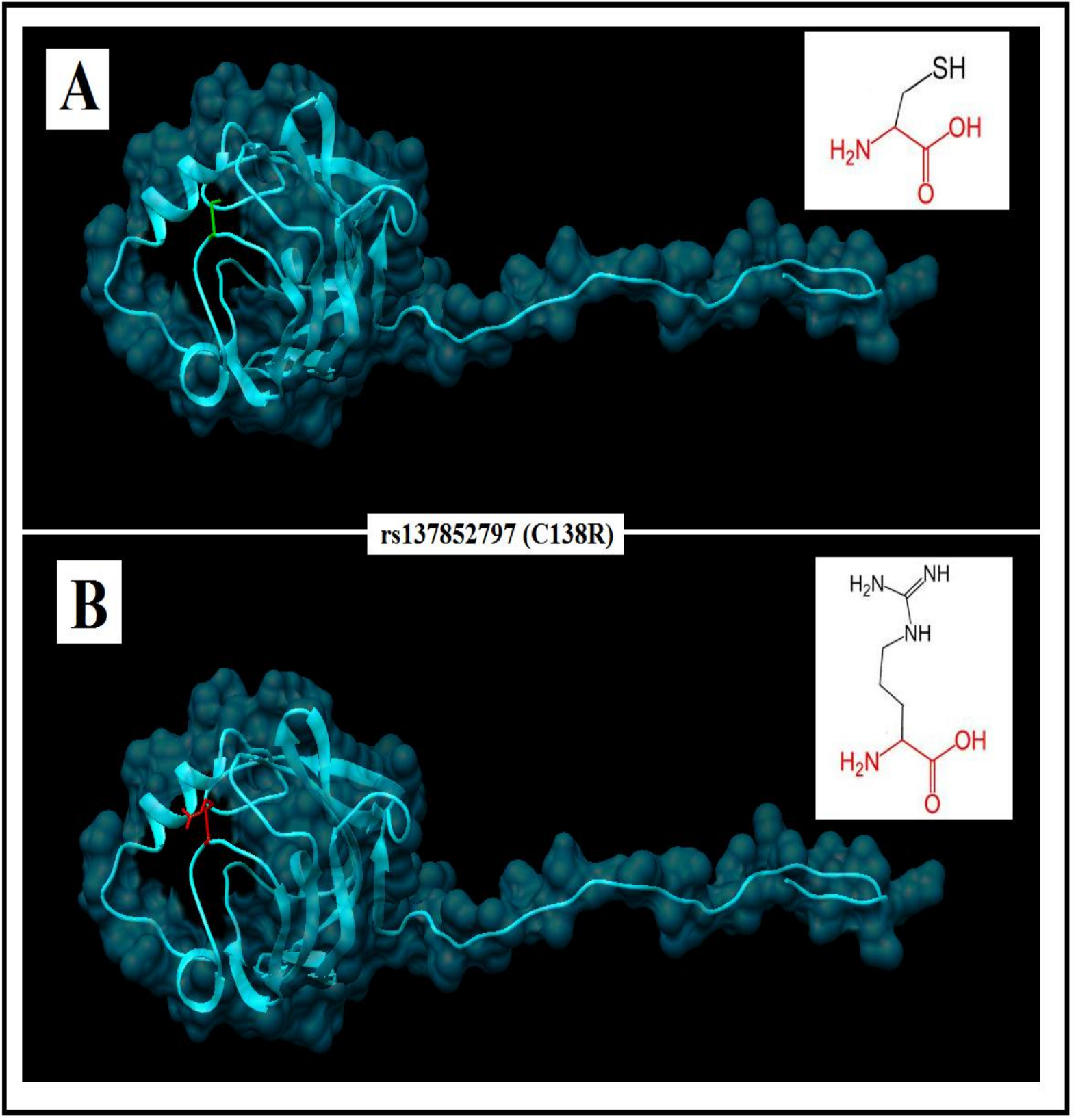

Figure (4) Effect of C138R (rs137852797) SNP on protein structure in which Cysteine mutated into Arginine at position 138 using project HOPE and Chimera. 


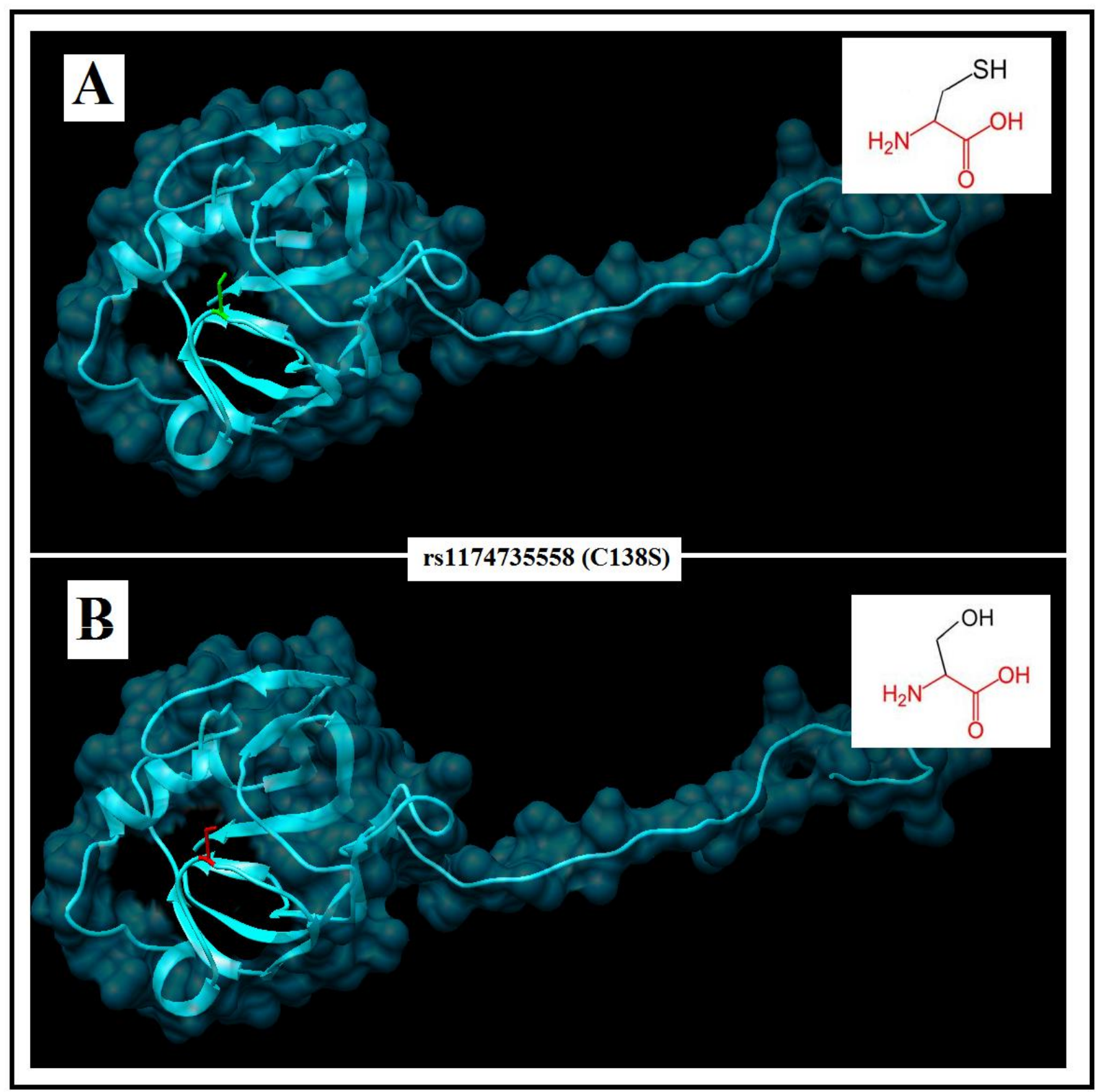

Figure (5) Effect of C138S (rs137852797) SNP on protein structure in which Cysteine mutated into Serine at position 138 using project HOPE and Chimera. 
3.6 SNPs effect on 3'UTR Region (miRNA binding sites) in GM2A using PolymiRTS Database

Table (4): prediction of SNPs sites in GM2A gene at the 3'UTR Region using PolymiRTS

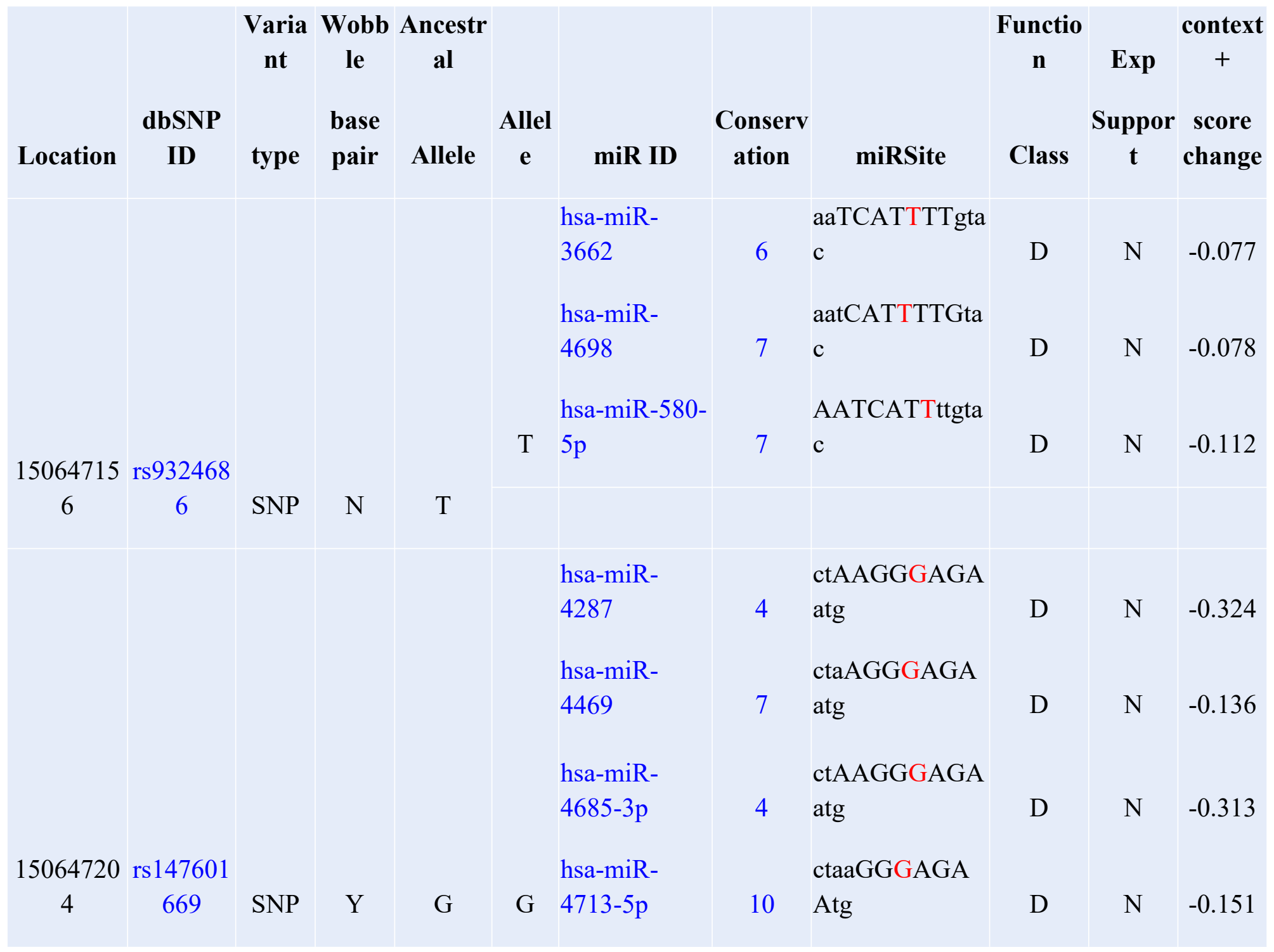




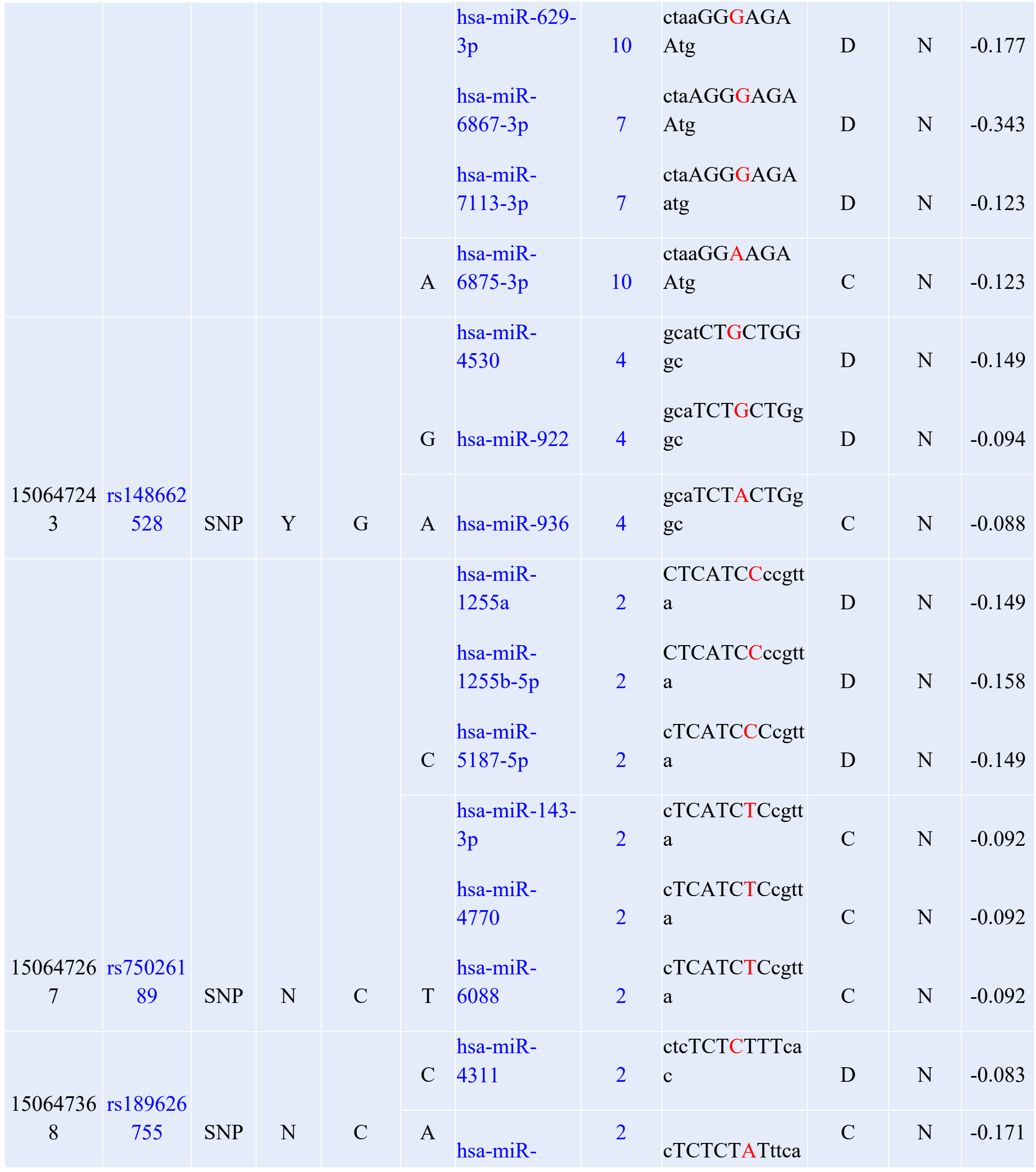


15064737 rs 104872

3

(3)
hsa-miR-

A $\quad$ T 4311

C

hsa-miR-

\begin{tabular}{l|l|l|l|l|l}
$548 \mathrm{az}-5 \mathrm{p}$ & 2 & $\mathrm{ttCACTTTTttt}$ & $\mathrm{O}$ & $\mathrm{N}$ & -0.097
\end{tabular}

hsa-miR-

15064737 rs201142 INDE

6

293

L N

15064738 rs 781088

8

78

SNP N

hsa-miR-

G 1304-3p

15064741 rs116809

\begin{tabular}{l|l|l|l|l}
3 & 628 & SNP & Y & G
\end{tabular}

15064742 rs 181890

2

739

SNP N

2 tctttcTCTCTTT

C

$\mathrm{N} \quad-0.115$

- 548t-5p

hsa-miR-

3613-3p

hsa-miR-

T 4504

7 ttttTTGTCACt

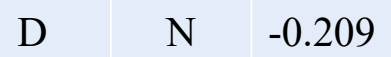

$\mathrm{T}$

aAGTGAGAgta

$4 \mathrm{tt}$

$\begin{array}{lll}\mathrm{D} & \mathrm{N} & -0.064\end{array}$

hsa-miR-424-

C $3 p$

2 tattaACGTTTTt

D

$\mathrm{N} \quad-0.133$

C

hsa-miR-

G $\quad 3130-3 p$

hsa-miR-

3923

hsa-miR-

4704-5p

15064754 rs 151210

\begin{tabular}{l|l|l|l|l|l}
8 & 034 & SNP & N & G & T
\end{tabular}

hsa-miR-
tactagGGTGCA

$2 \mathrm{G}$

D $\quad \mathrm{N} \quad-0.162$

TACTAGTgtgc

$\begin{array}{llllll}2 & \text { ag } & \text { C } & \text { N } & -0.168\end{array}$

taCTAGTGTgc

$\begin{array}{lllll}2 & \text { ag } & \text { C } & \text { N } & -0.147\end{array}$

$\begin{array}{llllll}2 & & \text { C } & \text { N } & -0.272\end{array}$ 


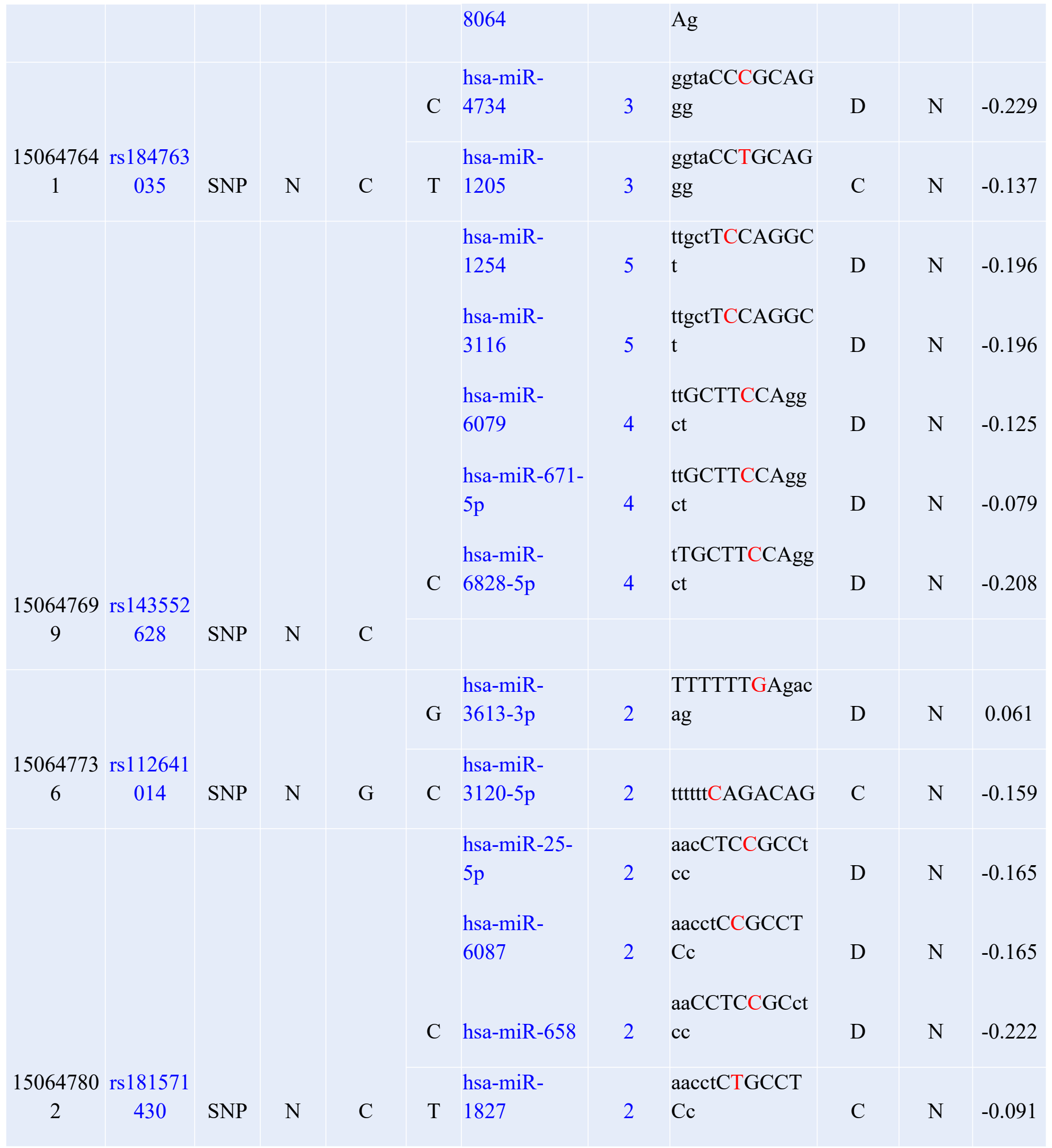




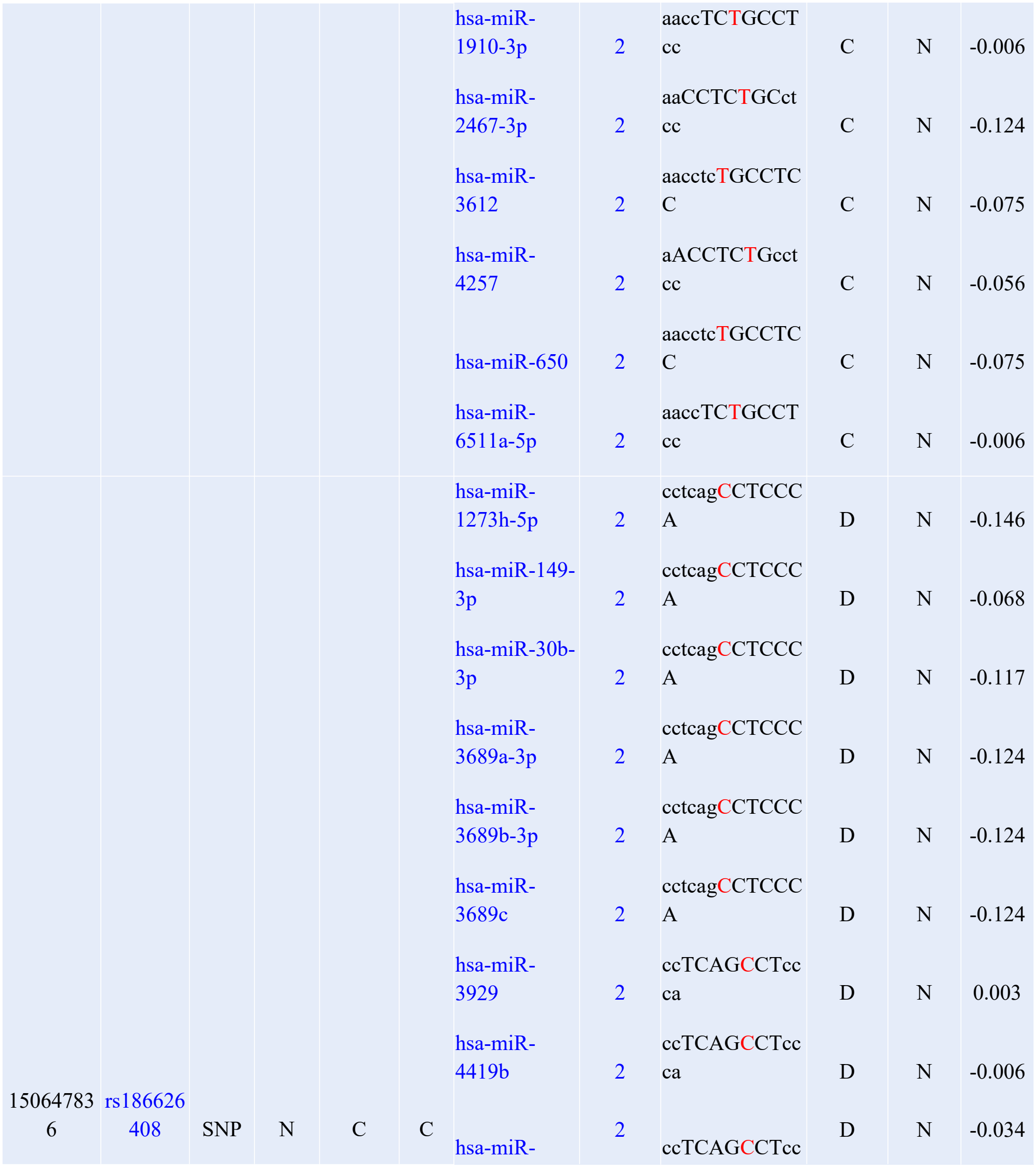




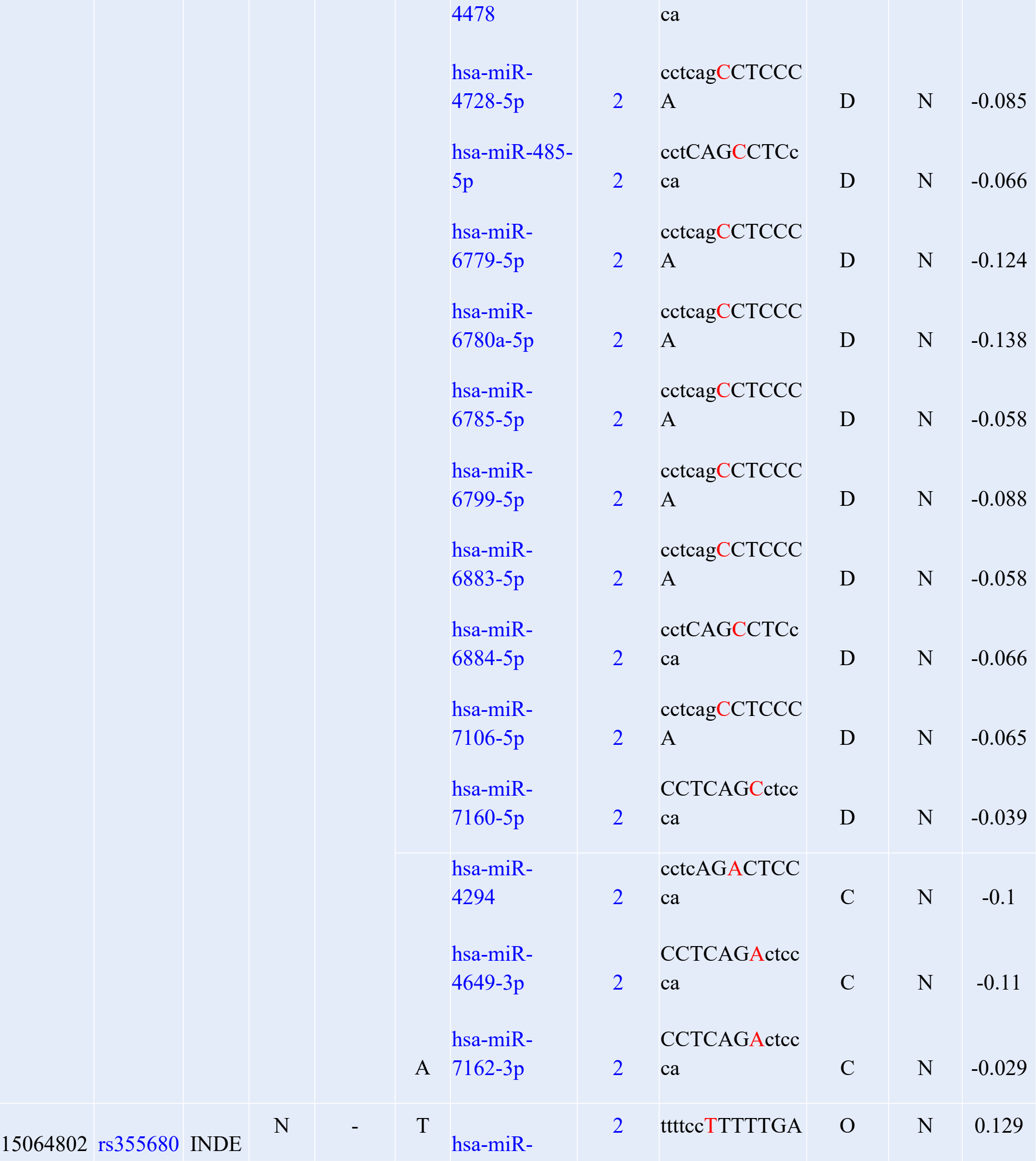




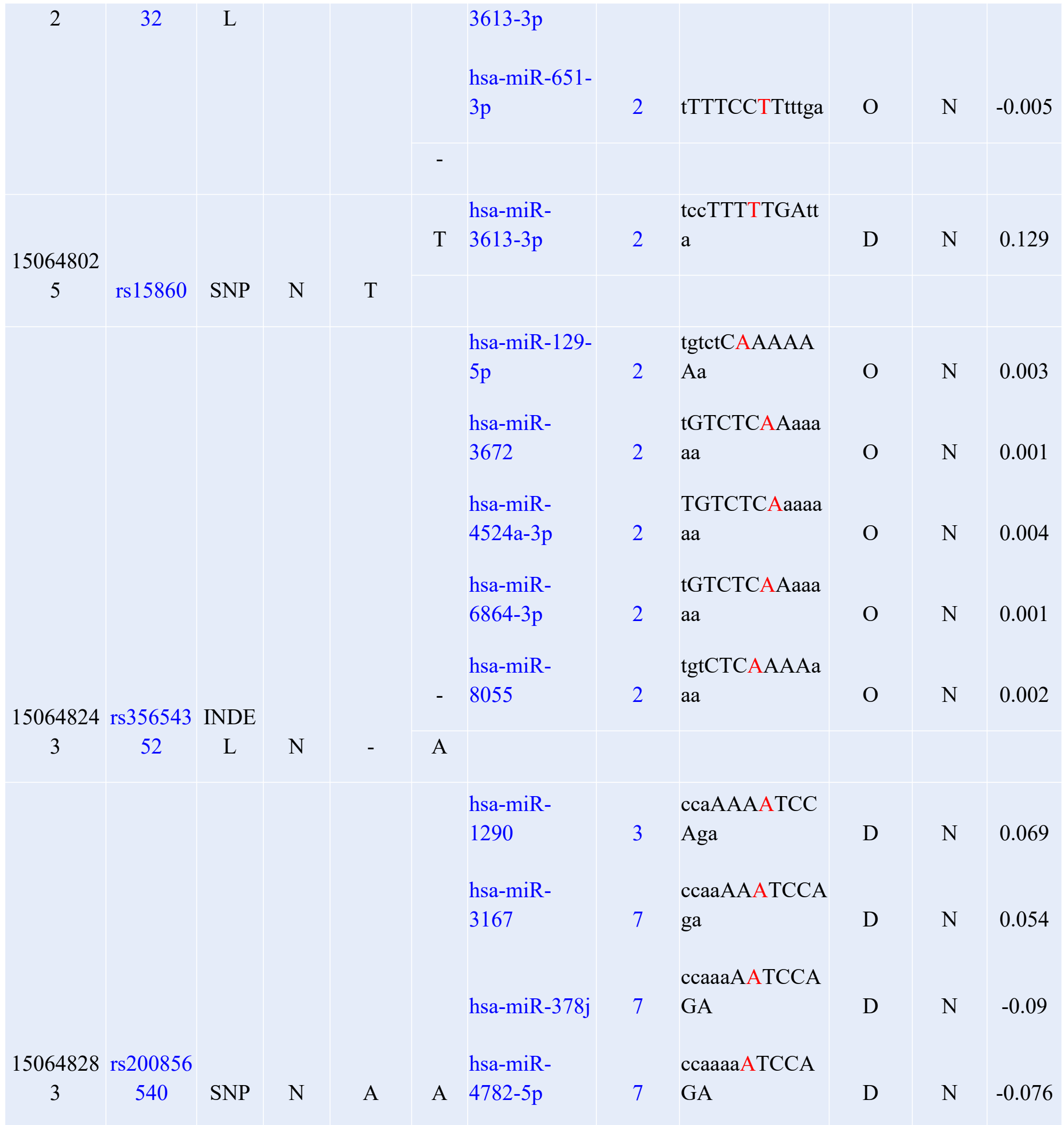




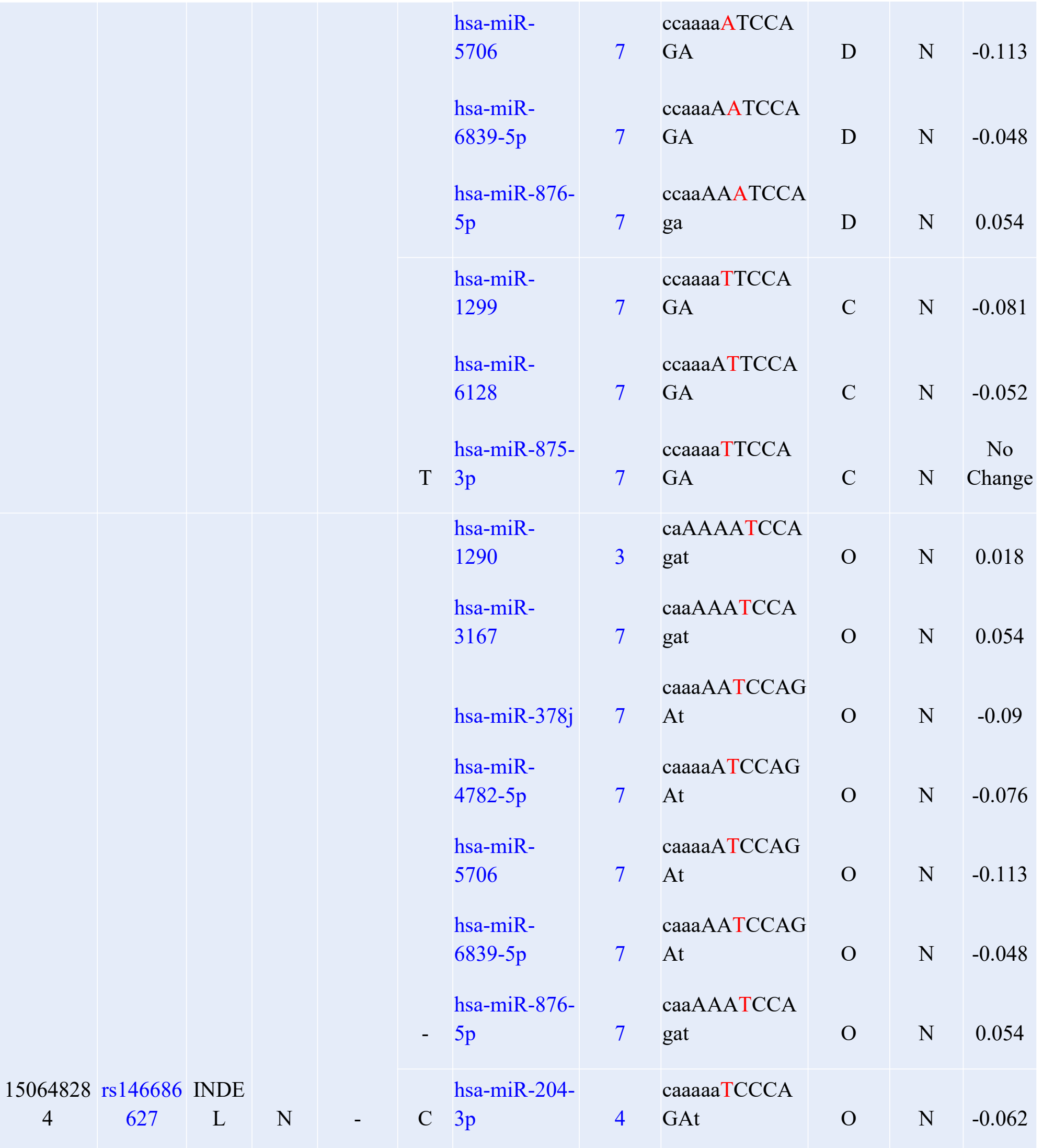




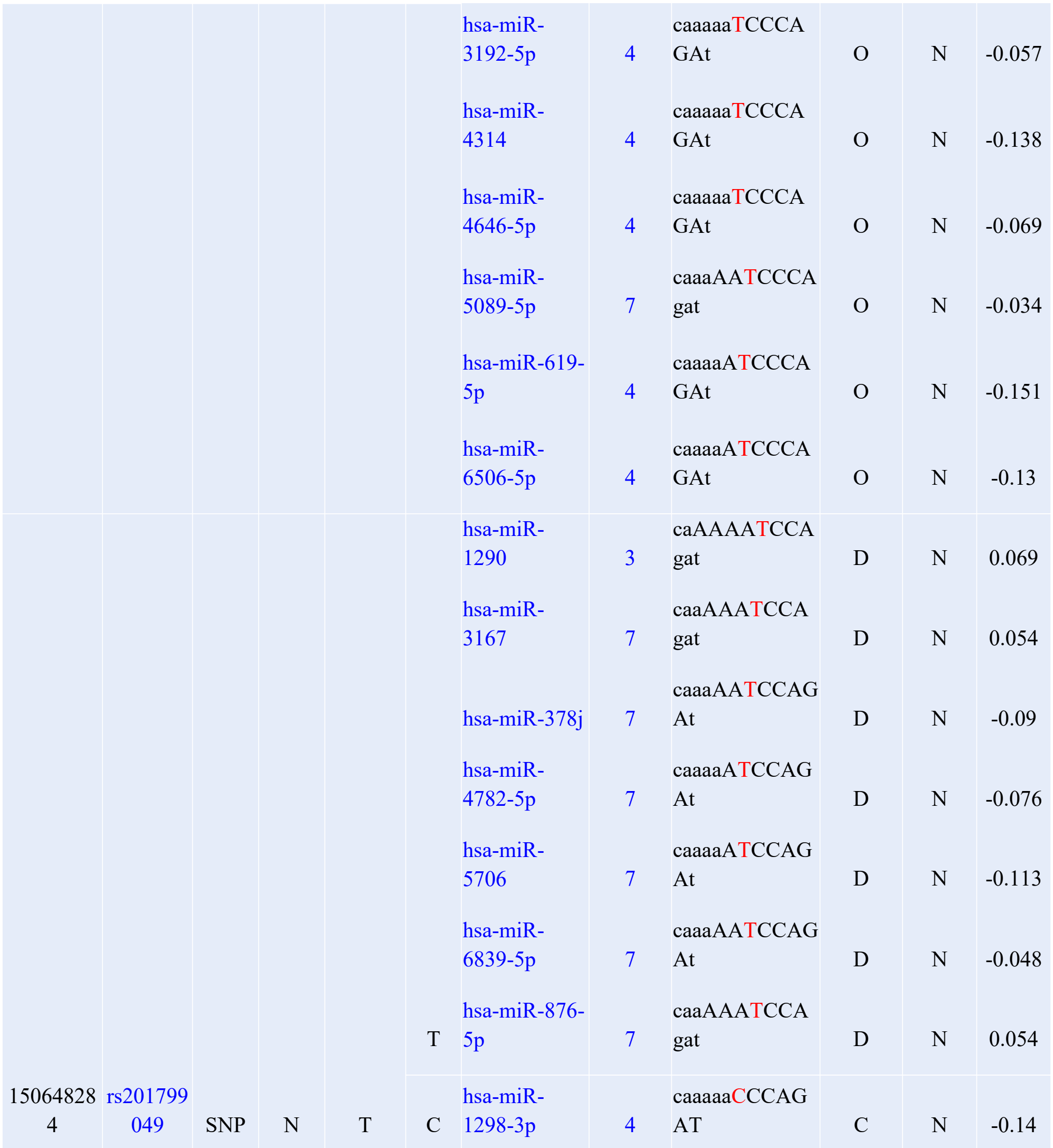




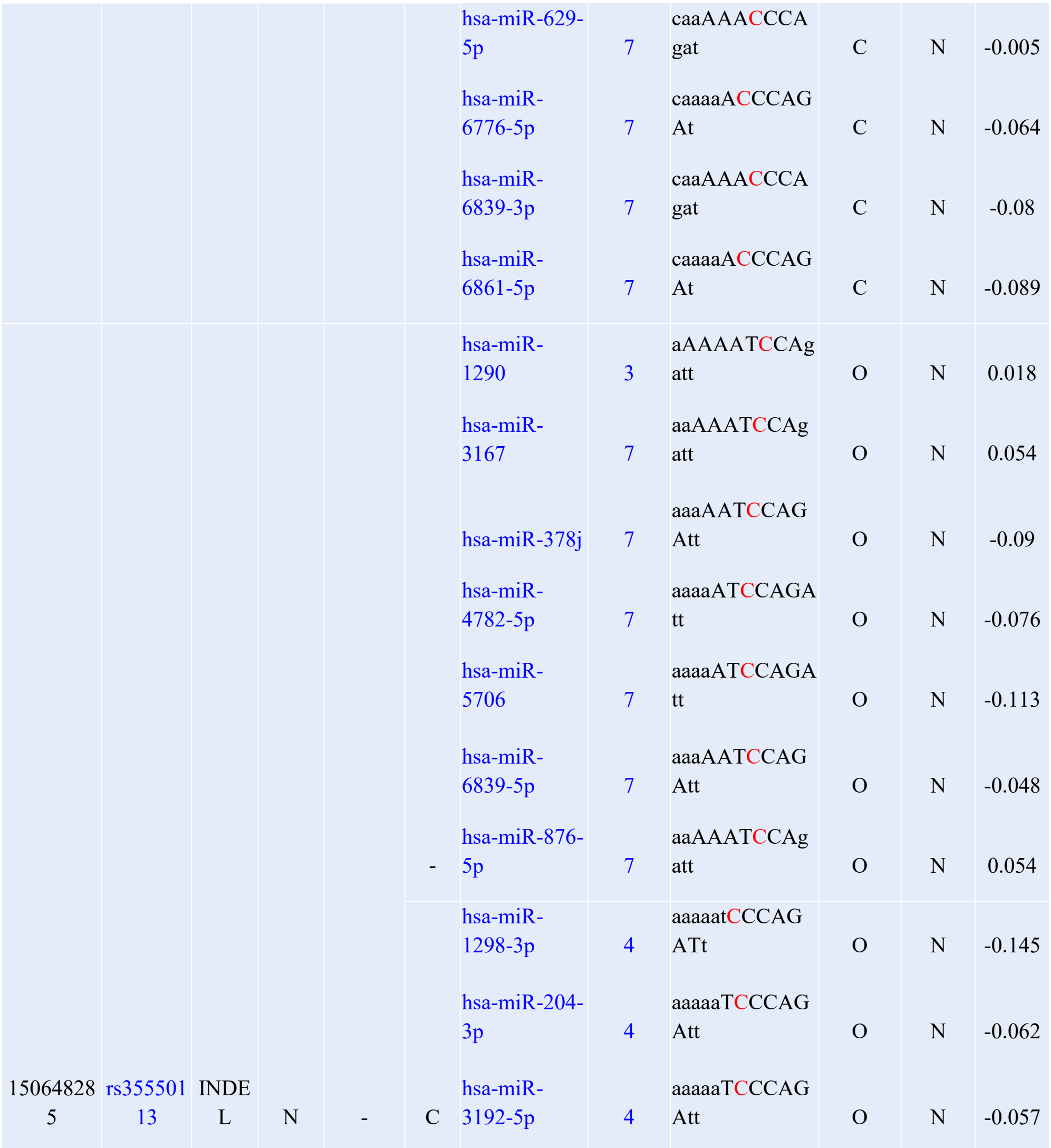




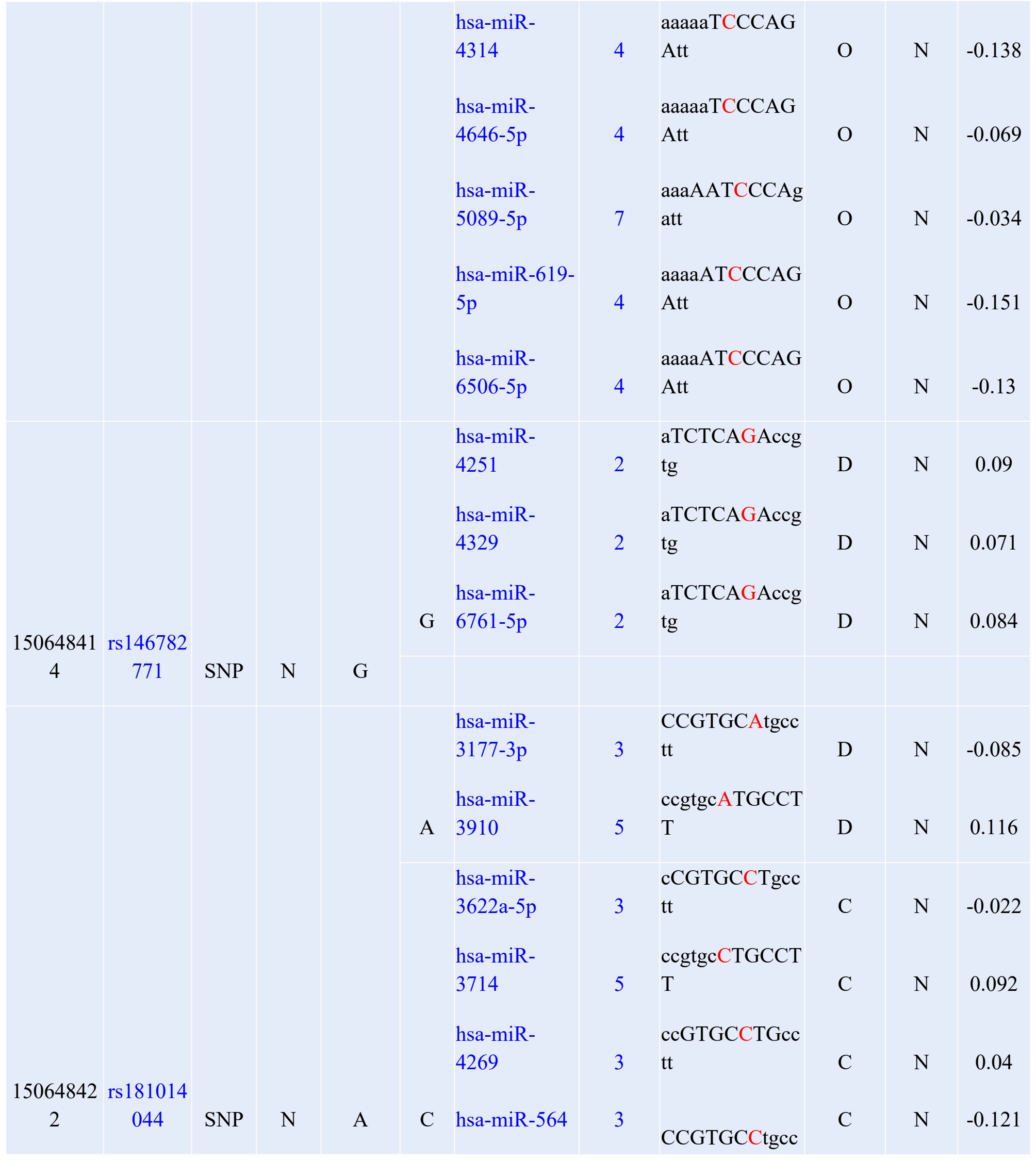




\begin{tabular}{|c|c|c|c|c|c|}
\hline $\begin{array}{l}\text { hsa-miR- } \\
6715 b-5 p\end{array}$ & 3 & $\begin{array}{l}\text { ccGTGCCTGcc } \\
\text { tt }\end{array}$ & $\mathrm{C}$ & $\mathrm{N}$ & 0.04 \\
\hline $\begin{array}{l}\text { hsa-miR- } \\
6808-5 p\end{array}$ & 3 & $\begin{array}{l}\text { ccgtgCCTGCC } \\
\mathrm{Tt}\end{array}$ & $\mathrm{C}$ & $\mathrm{N}$ & 0.028 \\
\hline $\begin{array}{l}\text { hsa-miR- } \\
6893-5 p\end{array}$ & 3 & $\begin{array}{l}\text { ccgtgCCTGCC } \\
\mathrm{Tt}\end{array}$ & $\mathrm{C}$ & $\mathrm{N}$ & 0.009 \\
\hline hsa-miR-940 & 3 & $\begin{array}{l}\text { ccgtgCCTGCC } \\
\mathrm{Tt}\end{array}$ & $\mathrm{C}$ & $\mathrm{N}$ & 0.037 \\
\hline
\end{tabular}

hsa-miR-539-

$\begin{array}{llllllll}7 & \text { rs } 153450 & \text { SNP } & \text { Y } & \text { G } & \text { A } & 5 p\end{array}$

hsa-miR-

1302

hsa-miR-

3122

hsa-miR-

3649

hsa-miR-

3913-5p

hsa-miR-

G 4298

hsa-miR-

1292-5p

hsa-miR-

4471

15064849

3
hsa-miR-

G $\quad$ T 450a-1-3p

ggcaccATTTCT

$3 \mathrm{C}$

tgtccgGTCCCA

2 A

$\begin{array}{lll}\text { D } & \mathrm{N} & -0.001\end{array}$

$\operatorname{tgtccgGTCCCA}$

2 A

$\begin{array}{lll}\mathrm{D} & \mathrm{N} & 0.076\end{array}$

tgtccGGTCCC

$\begin{array}{lllll}2 & \mathrm{Aa} & \mathrm{D} & \mathrm{N} & -0.051\end{array}$

$\operatorname{tgtccgGTCCCA}$

2 A

$\begin{array}{lll}\mathrm{D} & \mathrm{N} & 0.104\end{array}$

$\operatorname{tgtccgGTCCCA}$

2 A

D $\quad \mathrm{N} \quad 0.013$

tgtcCGTTCCC

2 Aa

$\begin{array}{lll}\mathrm{C} & \mathrm{N} & -0.051\end{array}$

tgtccGTTCCCA

$2 \mathrm{a}$

$\begin{array}{lll}\mathrm{C} & \mathrm{N} & 0.012\end{array}$

tgtccgTTCCCA

2 A

C N

0.161 


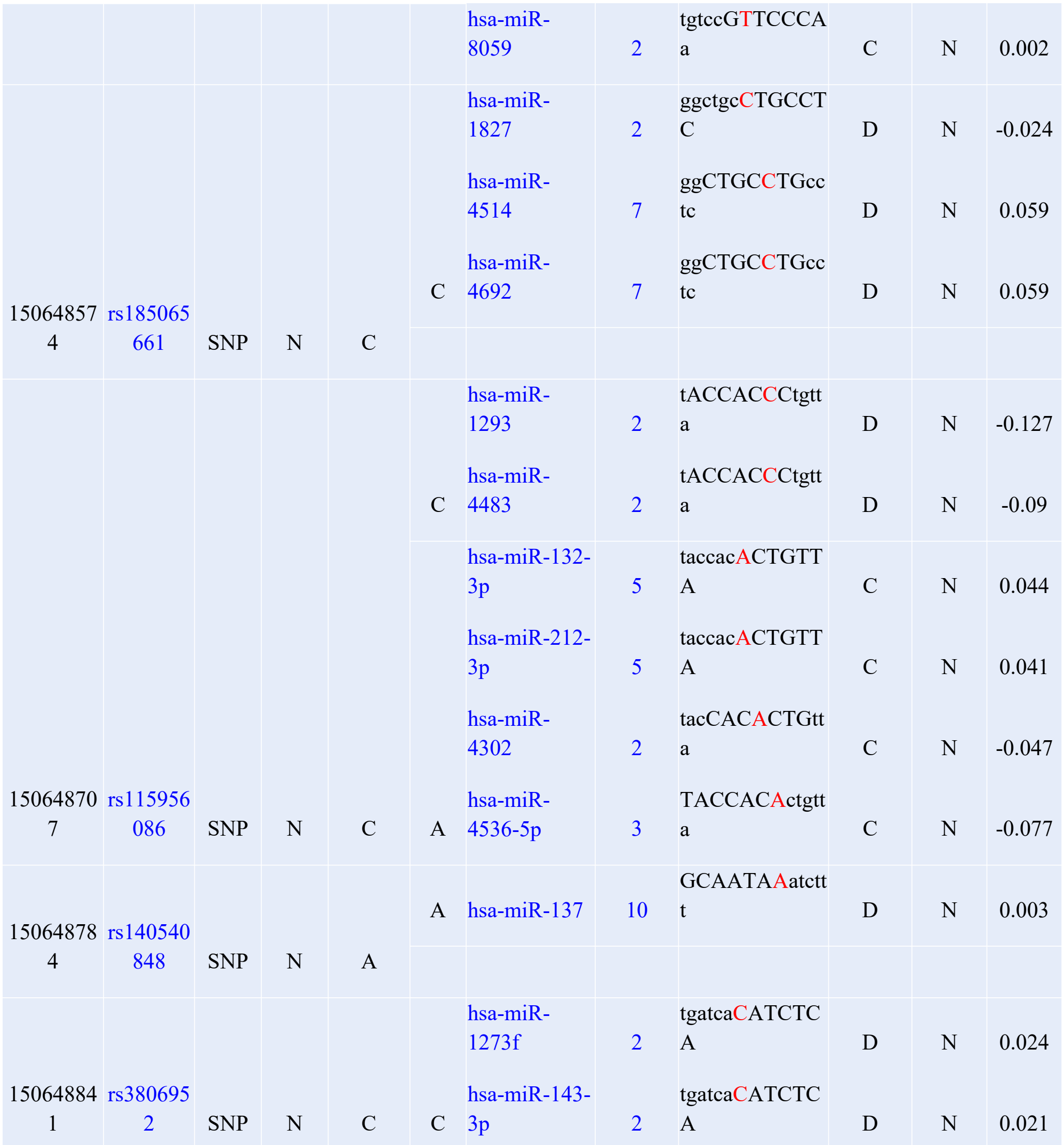




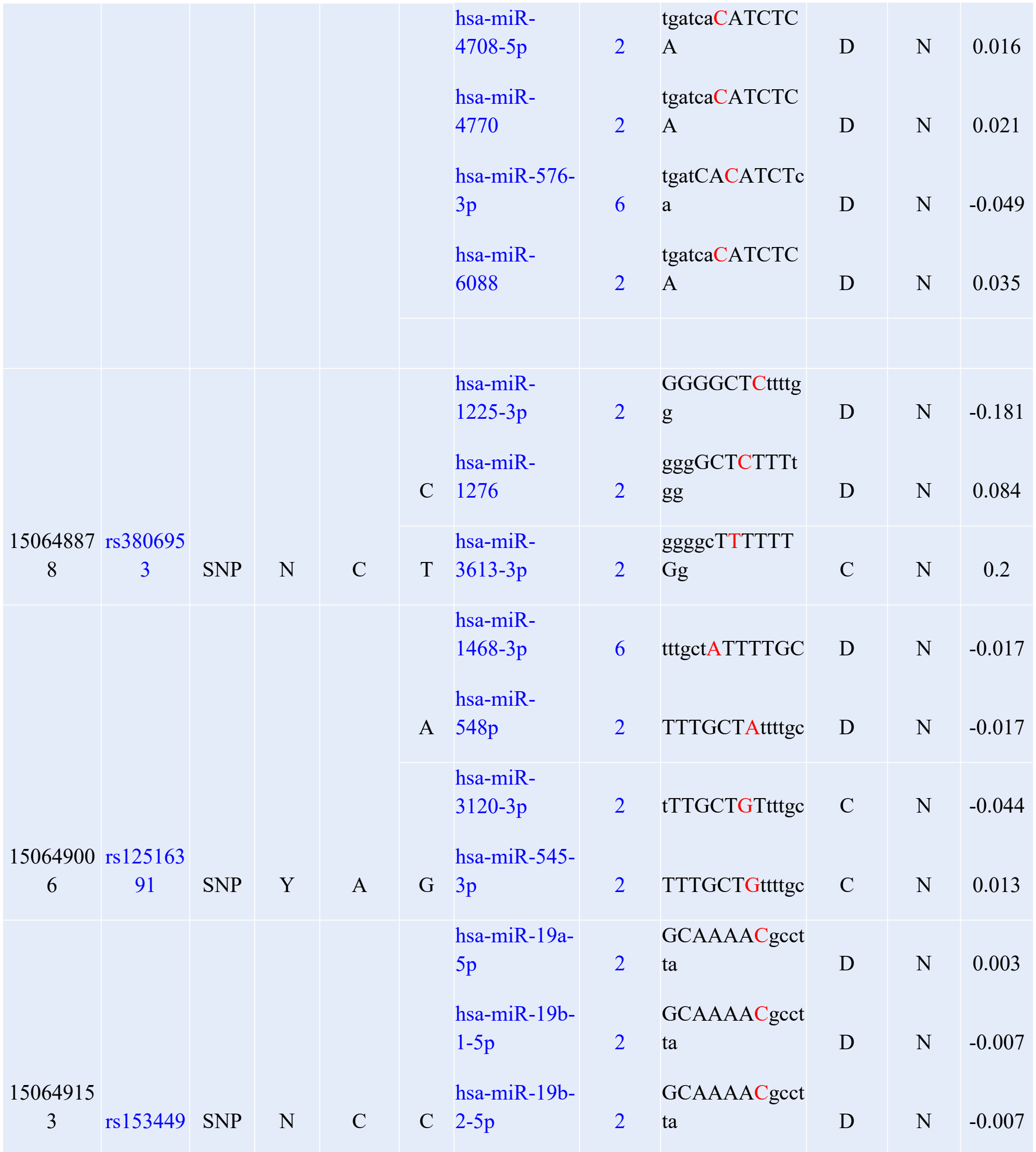




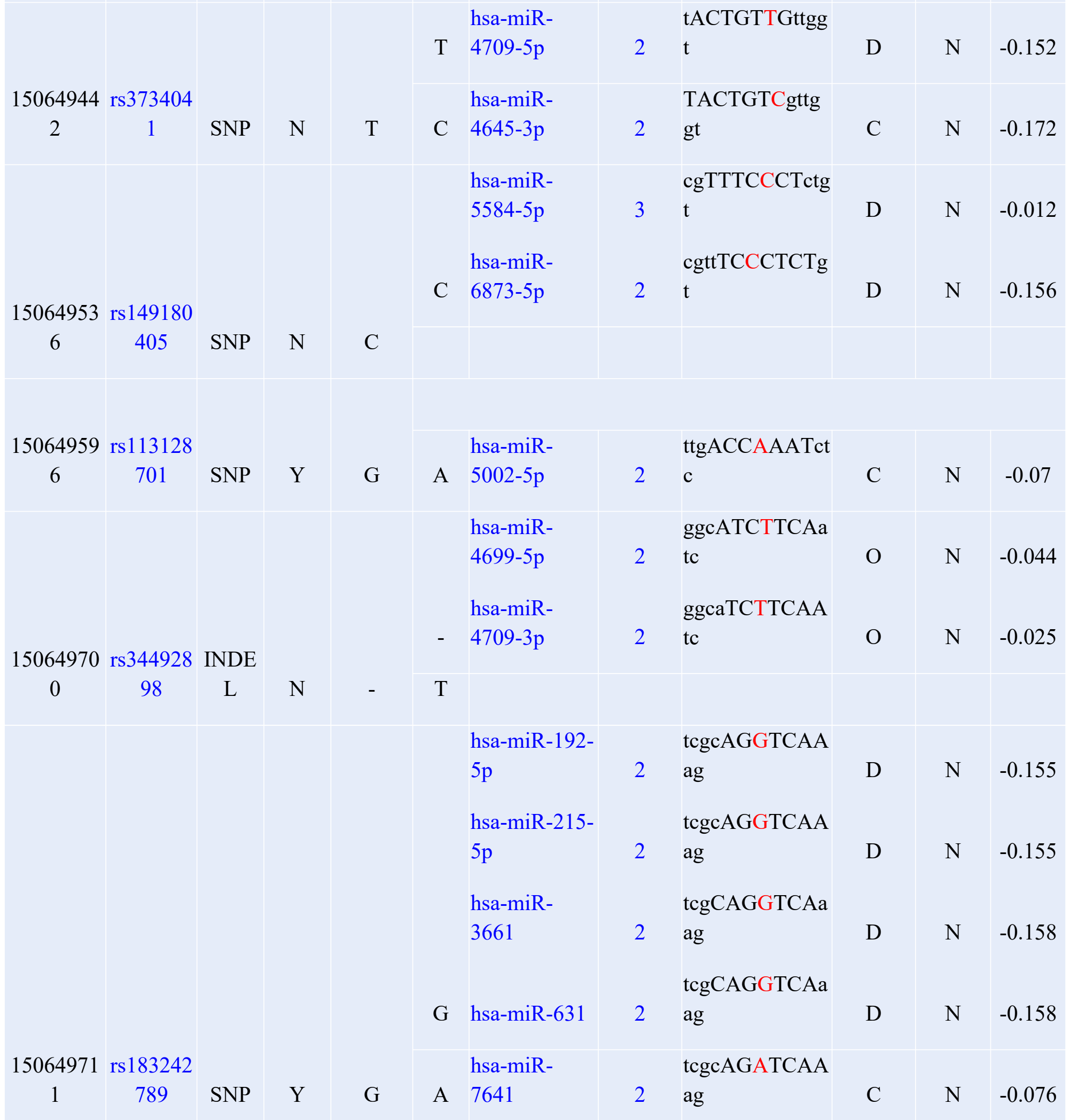




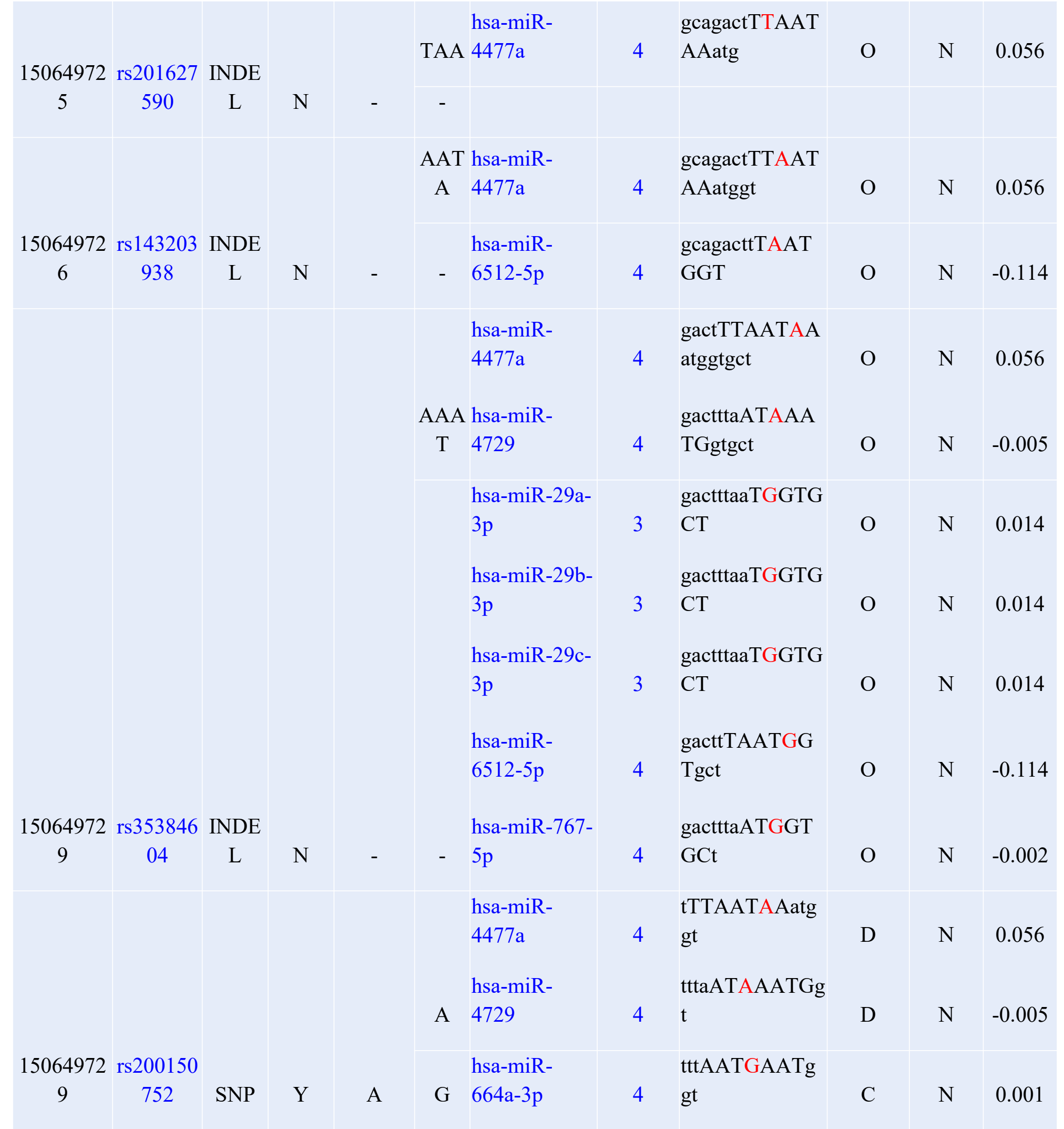




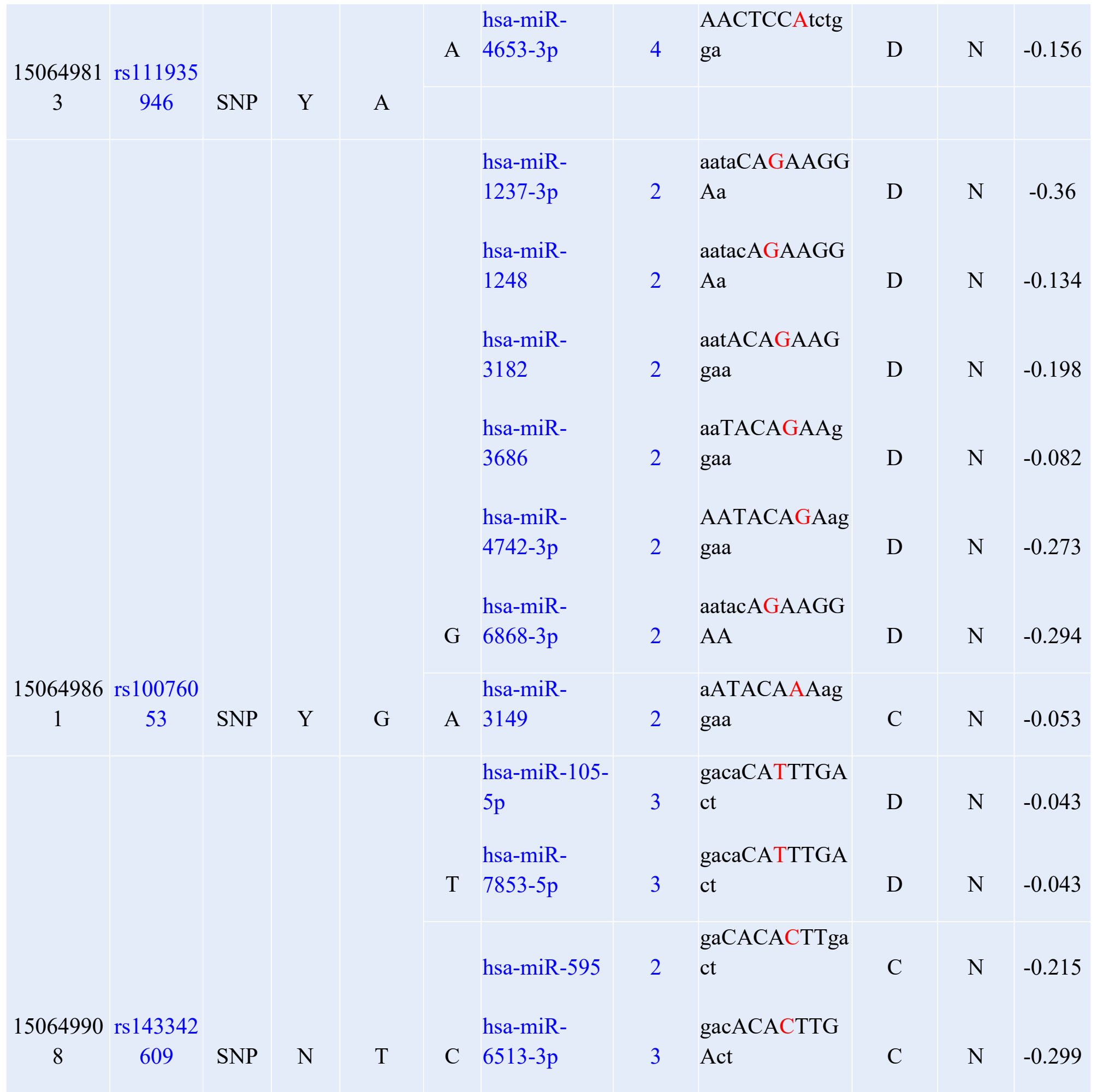




\section{Interactions of GM2Agene with other Functional Genes illustrated by GeneMANIA}

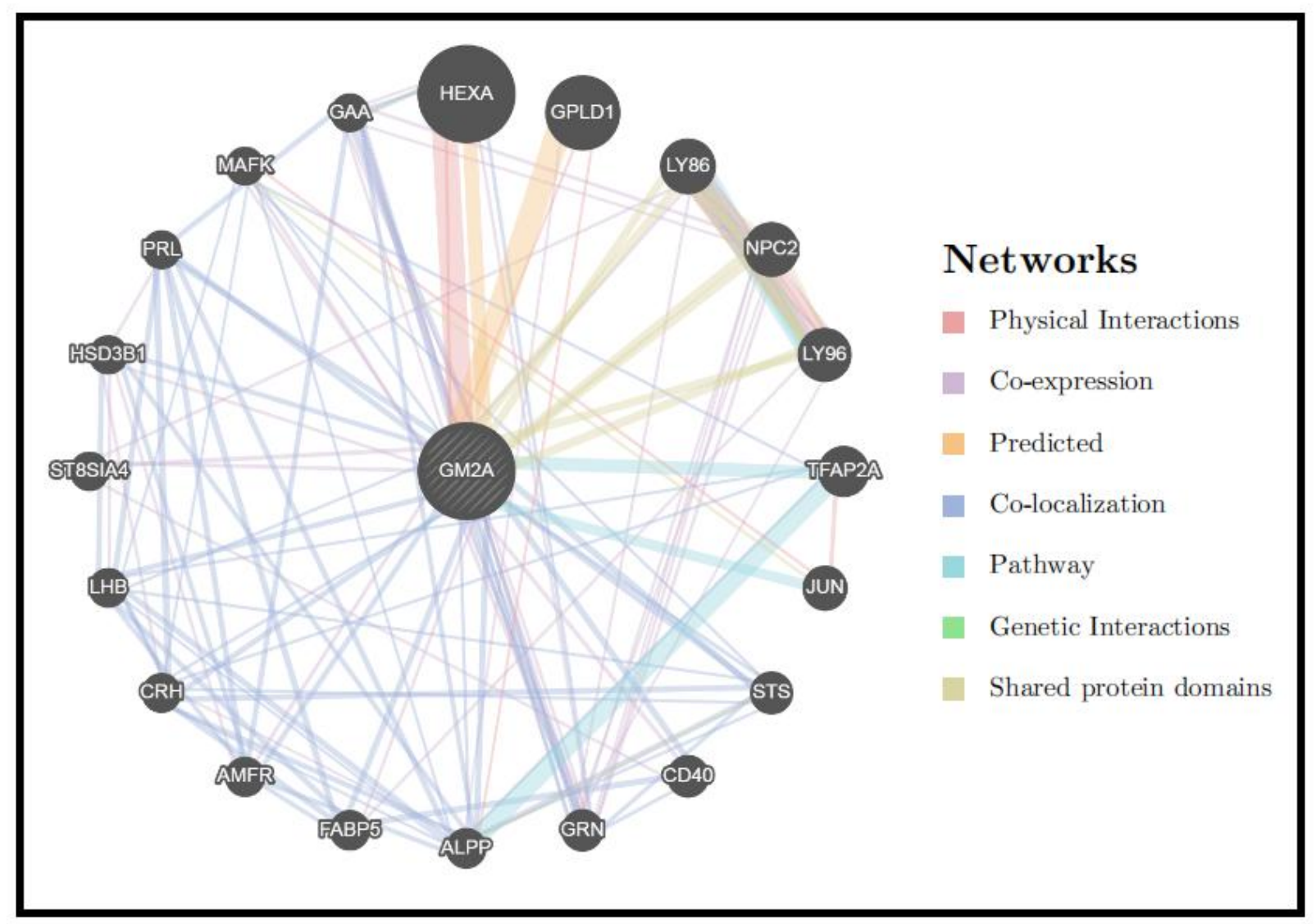

Figure 3: $G M 2 A$ Gene Interactions and network predicted by GeneMania.

Table (5): The $G M 2 A$ gene functions and its appearance in network and genome

\begin{tabular}{llll}
\hline Function & FDR & Genes in network & Genes in genome \\
\hline glycolipid metabolic process & 0.000729126 & 5 & 91 \\
membrane lipid metabolic process & 0.002107403 & 5 & 140 \\
glycosphingolipid metabolic process & 0.002107403 & 4 & 55 \\
sphingolipid metabolic process & 0.018998673 & 4 & 102 \\
regulation of hormone levels & 0.566018537 & 4 & 269 \\
vacuolar part & 0.566018537 & 4 & 262 \\
\hline
\end{tabular}


Table (6): The genes co-expressed, co localized, Physical Interacted and share a domain with IL2RG

\begin{tabular}{|c|c|c|c|c|}
\hline Gene 1 & Gene 2 & Weight & Network group & Network \\
\hline GAA & $N P C 2$ & 0.013373704 & Co-expression & Ramaswamy-Golub-2001 \\
\hline GAA & GRN & 0.009285928 & Co-expression & Ramaswamy-Golub-2001 \\
\hline NPC2 & LY86 & 0.006769352 & Co-expression & Wang-Maris-2006 \\
\hline LY96 & LY86 & 0.006558455 & Co-expression & Wang-Maris-2006 \\
\hline LY96 & NPC2 & 0.004556628 & Co-expression & Wang-Maris-2006 \\
\hline GRN & NPC2 & 0.006593361 & Co-expression & Wang-Maris-2006 \\
\hline MAFK & GM2A & 0.012908783 & Co-expression & Wang-Maris-2006 \\
\hline LY96 & $L Y 86$ & 0.009755008 & Co-expression & Mallon-McKay-2013 \\
\hline HSD3B1 & GM2A & 0.012744123 & Co-expression & Mallon-McKay-2013 \\
\hline HSD3B1 & CRH & 0.011914967 & Co-expression & Mallon-McKay-2013 \\
\hline HSD3B1 & $L H B$ & 0.011909817 & Co-expression & Mallon-McKay-2013 \\
\hline ST8SIA4 & GM2A & 0.02893034 & Co-expression & Bild-Nevins-2006 B \\
\hline \multirow[t]{2}{*}{ MAFK } & GM2A & 0.021602744 & Co-expression & Bild-Nevins-2006 B \\
\hline & & & & Burington-Shaughnessy- \\
\hline \multirow[t]{2}{*}{ GRN } & $G M 2 A$ & 0.020527184 & Co-expression & 2008 \\
\hline & & & & Burington-Shaughnessy- \\
\hline \multirow[t]{2}{*}{$A M F R$} & GM2A & 0.020826323 & Co-expression & 2008 \\
\hline & & & & Burington-Shaughnessy- \\
\hline \multirow[t]{2}{*}{ ST8SIA4 } & $C D 40$ & 0.01166518 & Co-expression & 2008 \\
\hline & & & & Burington-Shaughnessy- \\
\hline GAA & GRN & 0.021335509 & Co-expression & 2008 \\
\hline$C D 40$ & $G M 2 A$ & 0.026018724 & Co-expression & Dobbin-Giordano-2005 \\
\hline ST8SIA4 & $G M 2 A$ & 0.02396974 & Co-expression & Dobbin-Giordano-2005 \\
\hline
\end{tabular}




\begin{tabular}{|c|c|c|c|c|}
\hline ST8SIA4 & LY86 & 0.01011246 & Co-expression & Dobbin-Giordano-2005 \\
\hline LY86 & GM2A & 0.014090131 & Co-expression & Bahr-Bowler-2013 \\
\hline GRN & NPC2 & 0.003417451 & Co-expression & Bahr-Bowler-2013 \\
\hline GAA & NPC2 & 0.008345172 & Co-expression & Bahr-Bowler-2013 \\
\hline GAA & GRN & 0.007346965 & Co-expression & Bahr-Bowler-2013 \\
\hline LY96 & LY86 & 0.006099948 & Co-expression & Innocenti-Brown-2011 \\
\hline GRN & LY86 & 0.006802562 & Co-expression & Innocenti-Brown-2011 \\
\hline GRN & NPC2 & 0.01666259 & Co-expression & Innocenti-Brown-2011 \\
\hline FABP5 & LY96 & 0.011711845 & Co-expression & Innocenti-Brown-2011 \\
\hline LY96 & NPC2 & 0.006187806 & Co-expression & Rieger-Chu-2004 \\
\hline GRN & $G M 2 A$ & 0.010561576 & Co-expression & Rieger-Chu-2004 \\
\hline GRN & NPC2 & 0.005401576 & Co-expression & Rieger-Chu-2004 \\
\hline GRN & LY96 & 0.003754711 & Co-expression & Rieger-Chu-2004 \\
\hline FABP5 & $G M 2 A$ & 0.014316405 & Co-expression & Roth-Zlotnik-2006 \\
\hline$G A A$ & $G M 2 A$ & 0.010570861 & Co-expression & Perou-Botstein-2000 \\
\hline$A L P P$ & GPLD1 & 0.003473165 & Co-expression & Smirnov-Cheung-2009 \\
\hline $\mathrm{CRH}$ & $A L P P$ & 0.004937839 & Co-expression & Wang-Cheung-2015 \\
\hline GRN & HEXA & 0.01568475 & Co-expression & Chen-Brown-2002 \\
\hline$G R N$ & NPC2 & 0.016359169 & Co-expression & Chen-Brown-2002 \\
\hline HSD3B1 & $A M F R$ & 0.018177362 & Co-expression & Chen-Brown-2002 \\
\hline GAA & HEXA & 0.01394323 & Co-expression & Chen-Brown-2002 \\
\hline GAA & GRN & 0.012652976 & Co-expression & Chen-Brown-2002 \\
\hline$P R L$ & HSD3B1 & 0.019465497 & Co-expression & Wu-Garvey-2007 \\
\hline STS & $G M 2 A$ & 0.030206537 & Co-localization & Schadt-Shoemaker-2004 \\
\hline CD40 & GM2A & 0.028133877 & Co-localization & Schadt-Shoemaker-2004 \\
\hline
\end{tabular}




\begin{tabular}{|c|c|c|c|c|}
\hline GRN & GM2A & 0.014236832 & Co-localization & Schadt-Shoemaker-2004 \\
\hline GRN & HEXA & 0.009402739 & Co-localization & Schadt-Shoemaker-2004 \\
\hline GRN & STS & 0.013237921 & Co-localization & Schadt-Shoemaker-2004 \\
\hline GRN & CD4O & 0.008915158 & Co-localization & Schadt-Shoemaker-2004 \\
\hline$A L P P$ & GM2A & 0.025433496 & Co-localization & Schadt-Shoemaker-2004 \\
\hline$A L P P$ & STS & 0.025644204 & Co-localization & Schadt-Shoemaker-2004 \\
\hline FABP5 & GM2A & 0.02687657 & Co-localization & Schadt-Shoemaker-2004 \\
\hline FABP5 & CD4O & 0.027478961 & Co-localization & Schadt-Shoemaker-2004 \\
\hline$A M F R$ & $G M 2 A$ & 0.021205518 & Co-localization & Schadt-Shoemaker-2004 \\
\hline$A M F R$ & $A L P P$ & 0.017865056 & Co-localization & Schadt-Shoemaker-2004 \\
\hline$A M F R$ & FABP5 & 0.013939995 & Co-localization & Schadt-Shoemaker-2004 \\
\hline CRH & GM2A & 0.0229119 & Co-localization & Schadt-Shoemaker-2004 \\
\hline $\mathrm{CRH}$ & STS & 0.024494056 & Co-localization & Schadt-Shoemaker-2004 \\
\hline $\mathrm{CRH}$ & $A L P P$ & 0.020280294 & Co-localization & Schadt-Shoemaker-2004 \\
\hline CRH & FABP5 & 0.022545591 & Co-localization & Schadt-Shoemaker-2004 \\
\hline$\angle H B$ & $G M 2 A$ & 0.021348838 & Co-localization & Schadt-Shoemaker-2004 \\
\hline$L H B$ & $A L P P$ & 0.025315747 & Co-localization & Schadt-Shoemaker-2004 \\
\hline$L H B$ & FABP5 & 0.022165282 & Co-localization & Schadt-Shoemaker-2004 \\
\hline$\angle H B$ & $A M F R$ & 0.013310601 & Co-localization & Schadt-Shoemaker-2004 \\
\hline$L H B$ & $\mathrm{CRH}$ & 0.023500843 & Co-localization & Schadt-Shoemaker-2004 \\
\hline HSD3B1 & GM2A & 0.01956358 & Co-localization & Schadt-Shoemaker-2004 \\
\hline HSD3B1 & $A L P P$ & 0.018070487 & Co-localization & Schadt-Shoemaker-2004 \\
\hline HSD3B1 & AMFR & 0.017620761 & Co-localization & Schadt-Shoemaker-2004 \\
\hline HSD3B1 & $L H B$ & 0.021880075 & Co-localization & Schadt-Shoemaker-2004 \\
\hline$P R L$ & $G M 2 A$ & 0.026748382 & Co-localization & Schadt-Shoemaker-2004 \\
\hline
\end{tabular}




\begin{tabular}{|c|c|c|c|c|}
\hline$P R L$ & STS & 0.024578847 & Co-localization & Schadt-Shoemaker-2004 \\
\hline$P R L$ & $A L P P$ & 0.023220547 & Co-localization & Schadt-Shoemaker-2004 \\
\hline$P R L$ & FABP5 & 0.027938604 & Co-localization & Schadt-Shoemaker-2004 \\
\hline$P R L$ & $A M F R$ & 0.024674127 & Co-localization & Schadt-Shoemaker-2004 \\
\hline$P R L$ & $\mathrm{CRH}$ & 0.031325996 & Co-localization & Schadt-Shoemaker-2004 \\
\hline$P R L$ & $L H B$ & 0.024771485 & Co-localization & Schadt-Shoemaker-2004 \\
\hline GAA & $G M 2 A$ & 0.021490874 & Co-localization & Schadt-Shoemaker-2004 \\
\hline GAA & HEXA & 0.015766772 & Co-localization & Schadt-Shoemaker-2004 \\
\hline GAA & GRN & 0.011875112 & Co-localization & Schadt-Shoemaker-2004 \\
\hline GAA & $A L P P$ & 0.016947022 & Co-localization & Schadt-Shoemaker-2004 \\
\hline GAA & $A M F R$ & 0.021210423 & Co-localization & Schadt-Shoemaker-2004 \\
\hline GAA & $P R L$ & 0.022300243 & Co-localization & Schadt-Shoemaker-2004 \\
\hline LY96 & LY86 & 0.006775627 & Co-localization & Johnson-Shoemaker-2003 \\
\hline STS & $G M 2 A$ & 0.015290882 & Co-localization & Johnson-Shoemaker-2003 \\
\hline GRN & $G M 2 A$ & 0.017155321 & Co-localization & Johnson-Shoemaker-2003 \\
\hline$A L P P$ & $G M 2 A$ & 0.01002972 & Co-localization & Johnson-Shoemaker-2003 \\
\hline$A L P P$ & TFAP2A & 0.009357058 & Co-localization & Johnson-Shoemaker-2003 \\
\hline$A L P P$ & STS & 0.008613536 & Co-localization & Johnson-Shoemaker-2003 \\
\hline CRH & $G M 2 A$ & 0.008725192 & Co-localization & Johnson-Shoemaker-2003 \\
\hline $\mathrm{CRH}$ & TFAP2A & 0.008326863 & Co-localization & Johnson-Shoemaker-2003 \\
\hline CRH & STS & 0.007740852 & Co-localization & Johnson-Shoemaker-2003 \\
\hline $\mathrm{CRH}$ & $A L P P$ & 0.005179046 & Co-localization & Johnson-Shoemaker-2003 \\
\hline LHB & $G M 2 A$ & 0.008484612 & Co-localization & Johnson-Shoemaker-2003 \\
\hline$L H B$ & TFAP2A & 0.008305473 & Co-localization & Johnson-Shoemaker-2003 \\
\hline LHB & STS & 0.00752627 & Co-localization & Johnson-Shoemaker-2003 \\
\hline
\end{tabular}




\begin{tabular}{|c|c|c|c|c|}
\hline$L H B$ & $A L P P$ & 0.005130447 & Co-localization & Johnson-Shoemaker-2003 \\
\hline LHB & $\mathrm{CRH}$ & 0.004576681 & Co-localization & Johnson-Shoemaker-2003 \\
\hline MAFK & $G M 2 A$ & 0.010018589 & Co-localization & Johnson-Shoemaker-2003 \\
\hline MAFK & TFAP2A & 0.009307076 & Co-localization & Johnson-Shoemaker-2003 \\
\hline MAFK & STS & 0.007400996 & Co-localization & Johnson-Shoemaker-2003 \\
\hline MAFK & $A L P P$ & 0.005228889 & Co-localization & Johnson-Shoemaker-2003 \\
\hline MAFK & $C R H$ & 0.004663254 & Co-localization & Johnson-Shoemaker-2003 \\
\hline MAFK & $L H B$ & 0.004635511 & Co-localization & Johnson-Shoemaker-2003 \\
\hline GAA & HEXA & 0.014947882 & Co-localization & Johnson-Shoemaker-2003 \\
\hline GAA & GRN & 0.016629409 & Co-localization & Johnson-Shoemaker-2003 \\
\hline LY96 & LY86 & 0.1184115 & Pathway & Wu-Stein-2010 \\
\hline TFAP2A & GM2A & 0.082734935 & Pathway & Wu-Stein-2010 \\
\hline JUN & GM2A & 0.061802242 & Pathway & Wu-Stein-2010 \\
\hline$A L P P$ & TFAP2A & 0.12819351 & Pathway & Wu-Stein-2010 \\
\hline LY96 & LY86 & 0.1488301 & Pathway & REACTOME \\
\hline LY96 & LY86 & 0.70710677 & Physical Interactions & IREF-DIP \\
\hline JUN & TFAP2A & 0.069114916 & Physical Interactions & Li-Chen-2015 \\
\hline HEXA & $G M 2 A$ & 1 & Physical Interactions & $\begin{array}{l}\text { BIOGRID-SMALL-SCALE- } \\
\text { STUDIES }\end{array}$ \\
\hline MAFK & JUN & 0.03714322 & Physical Interactions & IREF-BIND \\
\hline HEXA & $G M 2 A$ & 0.77715117 & Physical Interactions & IREF-HPRD \\
\hline GPLD1 & $G M 2 A$ & 0.23492727 & Physical Interactions & IREF-HPRD \\
\hline ALPP & GPLD1 & 0.21245266 & Physical Interactions & IREF-HPRD \\
\hline HEXA & GM2A & 0.41421357 & Predicted & Wu-Stein-2010 \\
\hline GPLD1 & $G M 2 A$ & 0.76536685 & Predicted & Wu-Stein-2010 \\
\hline
\end{tabular}




\begin{tabular}{lllll} 
LY86 & GM2A & 0.1993388 & Shared protein domains & INTERPRO \\
NPC2 & GM2A & 0.1993388 & Shared protein domains & INTERPRO \\
NPC2 & LY86 & 0.054153882 & Shared protein domains & INTERPRO \\
LY96 & GM2A & 0.19933881 & Shared protein domains & INTERPRO \\
LY96 & LY86 & 0.054153886 & Shared protein domains & INTERPRO \\
LY96 & NPC2 & 0.054153886 & Shared protein domains & INTERPRO \\
ALPP & STS & 0.018784788 & Shared protein domains & INTERPRO \\
MAFK & JUN & 0.025053639 & Shared protein domains & INTERPRO \\
GAA & HEXA & 0.009137378 & Shared protein domains & INTERPRO \\
LY86 & GM2A & 0.33333334 & Shared protein domains & PFAM \\
NPC2 & GM2A & 0.33333334 & Shared protein domains & PFAM \\
NPC2 & LY86 & 0.33333334 & Shared protein domains & PFAM \\
LY96 & GM2A & 0.33333334 & Shared protein domains & PFAM \\
LY96 & LY86 & 0.33333334 & Shared protein domains & PFAM \\
LY96 & NPC2 & 0.33333334 & Shared & PFAM \\
\hline
\end{tabular}

\section{DISCUSSION:}

According to our analysis we found four SNPs in GM2A gene possibly regarded as highly damaging SNPs which they are: C99Y, C112F, C138S and C138R. All of them are novel SNPs except C138R which is reported in previous studies. Sheth et al (2016) and Kochumon et al (2017) studies are agrre with us that C138R found in GM2A gene and associated with AB variant of GM2 gangliosidosis $(22,45)$.

Regarding C99Y (rs751417546) SNP, it has differences between wild-type and mutant residue in size and hydrophobicity; according to size, mutant residue is bigger than the wild-type residue that is located on the surface of the protein so mutation of this residue can disturb interactions with other molecules or other parts of the protein. On other hand, the wild-type residue is more hydrophobic than the mutant residue and the mutation might cause loss of hydrophobic interactions with other molecules on the surface of the protein. In case of C112F (rs773743799) 
SNP, the wild type residue is bigger than mutant residue also it buried in the core of a domain so the differences between the wild and mutant residue might disturb the core structure of this domain. Concerning C138S (rs1174735558) SNP, the wild-type residue is more hydrophobic than the mutant residue. The mutation might cause loss of hydrophobic interactions with other molecules on the surface of the protein. The mutation is located in a region with known splice variants. A mutation to "Arginine" was found at this position as C138R (rs137852797) which has dissimilarities between wild-type and mutant amino acids in size, charge and hydrophobicity. As the wild-type residue charge was NEUTRAL, the mutant residue charge is POSITIVE. The mutation introduces a charge at this position; this can cause repulsion between the mutant residue and neighboring residues. And According to size, the mutant residue is bigger than the wild-type residue. The residue is located on the surface of the protein; mutation of this residue can disturb interactions with other molecules or other parts of the protein. On other hand, the wild-type residue is more hydrophobic than the mutant residue. The mutation might cause loss of hydrophobic interactions with other molecules on the surface of the protein.

The wild-type residues of the four SNPs are annotated to be involved in a cysteine bridge, which is important for stability of the protein, only cysteines can make this type of bonds. The mutation causes loss of this interaction and will have a severe effect on the 3D-structure of the protein. Together with loss of the cysteine bond, the differences between the old and new residue can cause destabilization of the structure. Also the wild-type residues are extremely conserved, based on conservation scores these mutations are probably damaging to the protein.

46 SNPs out of 676 SNPs were predicted to affect miRNAs binding sites on 3'UTR leading to abnormal expression of the resulting protein. 112 alleles disrupt a conserved miRNAs binding sites while 57 derived alleles creating a new binding site of miRNAs.

GM2A gene function and activities illustrated by GENEMANI as following: glycolipid metabolic process, glycosphingolipid metabolic process, membrane lipid metabolic process, sphingolipid metabolic process, vacuolar part.

As the great role of using bioinformatics tools, laboratory analysis accompanied with in vivo analysis remains highly recommended confirming our findings. These findings could be used in a helpful way to improve the diagnosis and screening of AB variant of GM2 gangliosidosis. 


\section{CONCLUSION:}

Through using different bioinformatics tools, four SNPs were found to be highly damaging SNPs that affect function, structure and stability of $G M 2 A$ protein, which they are: $\mathrm{C} 99 \mathrm{Y}, \mathrm{C} 112 \mathrm{~F}$, C138S and C138R, three of them are novel SNPs (C99Y, C112F and C138S). Also 46 SNPs were predicted to affect miRNAs binding sites on 3'UTR leading to abnormal expression of the resulting protein. 112 alleles disrupt a conserved miRNAs binding sites while 57 derived alleles creating a new binding site of miRNAs. These SNPs should be considered as important candidates in causing $\mathrm{AB}$ variant of GM2 gangliosidosis and may help in diagnosis and genetic screening of the disease.

\section{ACKNOWLEDGMENT:}

The authors desire to acknowledge the exciting collaboration of Africa City of Technology Sudan.

\section{DATA AVAILABILITY:}

All relevant data used to support the findings of this study are included within the manuscript and supplementary information files.

\section{CONFLICT OF INTEREST:}

The authors declare that there is no conflict of interest regarding the publication of this paper.

\section{References:}

1. Bertoni C, Appolloni MG, Stirling JL, Li SC, Li YT, Orlacchio A, et al. Structural organization and expression of the gene for the mouse GM2 activator protein. Mammalian genome : official journal of the International Mammalian Genome Society. 1997;8(2):90-3.

2. Brackmann F, Kehrer C, Kustermann W, Bohringer J, Krageloh-Mann I, Trollmann R. Rare Variant of GM2 Gangliosidosis through Activator-Protein Deficiency. Neuropediatrics. 2017;48(2):127-30.

3. Conzelmann E, Sandhoff K, Nehrkorn H, Geiger B, Arnon R. Purification, biochemical and immunological characterisation of hexosaminidase $A$ from variant $A B$ of infantile $G M 2$ gangliosidosis. European journal of biochemistry. 1978;84(1):27-33.

4. Purpura DP, Suzuki K. Distortion of neuronal geometry and formation of aberrant synapses in neuronal storage disease. Brain research. 1976;116(1):1-21.

5. Conzelmann E, Sandhoff K. AB variant of infantile GM2 gangliosidosis: deficiency of a factor necessary for stimulation of hexosaminidase A-catalyzed degradation of ganglioside GM2 and glycolipid 
GA2. Proceedings of the National Academy of Sciences of the United States of America. 1978;75(8):3979-83.

6. Kytzia HJ, Hinrichs U, Maire I, Suzuki K, Sandhoff K. Variant of GM2-gangliosidosis with hexosaminidase A having a severely changed substrate specificity. The EMBO journal. 1983;2(7):1201-5. 7. Sandhoff K, Conzelmann E, Nehrkorn H. Substrate specificity of hexosaminidase A isolated from the liver of a patient with a rare form ( $A B$ variant) of infantile GM2 gangliosidosis and control tissues. Advances in experimental medicine and biology. 1978;101:727-30.

8. Cordeiro $P$, Hechtman $P$, Kaplan $F$. The GM2 gangliosidoses databases: allelic variation at the HEXA, HEXB, and GM2A gene loci. Genetics in medicine : official journal of the American College of Medical Genetics. 2000;2(6):319-27.

9. Hou Y, McInnes B, Hinek A, Karpati G, Mahuran D. A Pro504 --> Ser substitution in the betasubunit of beta-hexosaminidase $A$ inhibits alpha-subunit hydrolysis of $G M 2$ ganglioside, resulting in chronic Sandhoff disease. The Journal of biological chemistry. 1998;273(33):21386-92.

10. Mahuran DJ. Biochemical consequences of mutations causing the GM2 gangliosidoses. Biochimica et biophysica acta. 1999;1455(2-3):105-38.

11. Mahuran DJ. The GM2 activator protein, its roles as a co-factor in GM2 hydrolysis and as a general glycolipid transport protein. Biochimica et biophysica acta. 1998;1393(1):1-18.

12. Heng $\mathrm{HH}$, Xie B, Shi XM, Tsui LC, Mahuran DJ. Refined mapping of the GM2 activator protein (GM2A) locus to 5q31.3-q33.1, distal to the spinal muscular atrophy locus. Genomics. 1993;18(2):429-31. 13. Suzuki Y. [Disorders of sphingolipid activator proteins]. Nihon rinsho Japanese journal of clinical medicine. 1995;53(12):3025-7.

14. Swallow DM, Islam I, Fox MF, Povey S, Klima H, Schepers U, et al. Regional localization of the gene coding for the GM2 activator protein (GM2A) to chromosome 5q32-33 and confirmation of the assignment of GM2AP to chromosome 3. Annals of human genetics. 1993;57(3):187-93.

15. Liu Y, Hoffmann A, Grinberg A, Westphal H, McDonald MP, Miller KM, et al. Mouse model of GM2 activator deficiency manifests cerebellar pathology and motor impairment. Proceedings of the National Academy of Sciences of the United States of America. 1997;94(15):8138-43.

16. Li SC, Hirabayashi Y, Li YT. A new variant of type-AB GM2-gangliosidosis. Biochemical and biophysical research communications. 1981;101(2):479-85.

17. Chen B, Rigat B, Curry C, Mahuran DJ. Structure of the GM2A gene: identification of an exon 2 nonsense mutation and a naturally occurring transcript with an in-frame deletion of exon 2 . American journal of human genetics. 1999;65(1):77-87.

18. Martins C, Brunel-Guitton C, Lortie A, Gauvin F, Morales CR, Mitchell GA, et al. Atypical juvenile presentation of $\mathrm{GM} 2$ gangliosidosis $A B$ in a patient compound-heterozygote for c.259G > T and c.164C > T mutations in the GM2A gene. Molecular genetics and metabolism reports. 2017;11:24-9.

19. Renaud D, Brodsky M. GM2-Gangliosidosis, AB Variant: Clinical, Ophthalmological, MRI, and Molecular Findings. JIMD reports. 2016;25:83-6.

20. Schepers U, Glombitza G, Lemm T, Hoffmann A, Chabas A, Ozand P, et al. Molecular analysis of a GM2-activator deficiency in two patients with GM2-gangliosidosis $A B$ variant. American journal of human genetics. 1996;59(5):1048-56.

21. Schroder M, Schnabel D, Suzuki K, Sandhoff K. A mutation in the gene of a glycolipid-binding protein (GM2 activator) that causes GM2-gangliosidosis variant AB. FEBS letters. 1991;290(1-2):1-3.

22. Sheth J, Datar C, Mistri M, Bhavsar R, Sheth F, Shah K. GM2 gangliosidosis AB variant: novel mutation from India - a case report with a review. BMC Pediatr. 2016;16:88.

23. Salih MA, Seidahmed MZ, El Khashab HY, Hamad MH, Bosley TM, Burn S, et al. Mutation in GM2A Leads to a Progressive Chorea-dementia Syndrome. Tremor and other hyperkinetic movements (New York, NY). 2015;5:306. 
24. Tsuji D, Higashine Y, Matsuoka K, Sakuraba H, Itoh K. Therapeutic evaluation of GM2 gangliosidoses by ELISA using anti-GM2 ganglioside antibodies. Clinica chimica acta; international journal of clinical chemistry. 2007;378(1-2):38-41.

25. Pullarkat RK, Reha H, Beratis NG. Accumulation of ganglioside $\mathrm{Gm} 2$ in cerebrospinal fluid of a patient with the variant AB of infantile Gm2 gangliosidosis. Pediatrics. 1981;68(1):106-8.

26. Sandhoff $\mathrm{K}$, Christomanou $\mathrm{H}$. Biochemistry and genetics of gangliosidoses. Human genetics. 1979;50(2):107-43.

27. Sonderfeld S, Brendler S, Sandhoff K, Galjaard H, Hoogeveen AT. Genetic complementation in somatic cell hybrids of four variants of infantile GM2 gangliosidosis. Human genetics. 1985;71(3):196200.

28. Sonderfeld S, Conzelmann E, Schwarzmann G, Burg J, Hinrichs U, Sandhoff K. Incorporation and metabolism of ganglioside GM2 in skin fibroblasts from normal and GM2 gangliosidosis subjects. European journal of biochemistry. 1985;149(2):247-55.

29. Sakuraba H, Itoh K, Shimmoto M, Utsumi K, Kase R, Hashimoto Y, et al. GM2 gangliosidosis AB variant: clinical and biochemical studies of a Japanese patient. Neurology. 1999;52(2):372-7.

30. George Priya Doss C, Sudandiradoss C, Rajasekaran R, Choudhury P, Sinha P, Hota P, et al. Applications of computational algorithm tools to identify functional SNPs. Functional \& integrative genomics. 2008;8(4):309-16.

31. Benson DA, Cavanaugh M, Clark K, Karsch-Mizrachi I, Lipman DJ, Ostell J, et al. GenBank. Nucleic acids research. 2017;45(D1):D37-d42.

32. Artimo P, Jonnalagedda M, Arnold K, Baratin D, Csardi G, de Castro E, et al. ExPASy: SIB bioinformatics resource portal. Nucleic acids research. 2012;40(Web Server issue):W597-603.

33. Sim NL, Kumar P, Hu J, Henikoff S, Schneider G, Ng PC. SIFT web server: predicting effects of amino acid substitutions on proteins. Nucleic Acids Res. 2012;40(Web Server issue):W452-7.

34. Adzhubei IA, Schmidt S, Peshkin L, Ramensky VE, Gerasimova A, Bork P, et al. A method and server for predicting damaging missense mutations. Nature methods. 2010;7(4):248-9.

35. Choi Y, Sims GE, Murphy S, Miller JR, Chan AP. Predicting the functional effect of amino acid substitutions and indels. PloS one. 2012;7(10):e46688.

36. Hecht $\mathrm{M}$, Bromberg $\mathrm{Y}$, Rost $\mathrm{B}$. Better prediction of functional effects for sequence variants. BMC genomics. 2015;16 Suppl 8:S1.

37. Calabrese R, Capriotti E, Fariselli P, Martelli PL, Casadio R. Functional annotations improve the predictive score of human disease-related mutations in proteins. Hum Mutat. 2009;30(8):1237-44.

38. Lopez-Ferrando V, Gazzo A, de la Cruz X, Orozco M, Gelpi JL. PMut: a web-based tool for the annotation of pathological variants on proteins, 2017 update. Nucleic Acids Res. 2017;45(W1):W222-W8.

39. Capriotti E, Fariselli P, Casadio R. I-Mutant2.0: predicting stability changes upon mutation from the protein sequence or structure. Nucleic Acids Res. 2005;33(Web Server issue):W306-10.

40. Venselaar H, Te Beek TA, Kuipers RK, Hekkelman ML, Vriend G. Protein structure analysis of mutations causing inheritable diseases. An e-Science approach with life scientist friendly interfaces. BMC Bioinformatics. 2010;11:548.

41. Pettersen EF, Goddard TD, Huang CC, Couch GS, Greenblatt DM, Meng EC, et al. UCSF Chimera-a visualization system for exploratory research and analysis. J Comput Chem. 2004;25(13):1605-12.

42. Bhattacharya A, Ziebarth JD, Cui Y. PolymiRTS Database 3.0: linking polymorphisms in microRNAs and their target sites with human diseases and biological pathways. Nucleic Acids Res. 2014;42(Database issue):D86-91.

43. Kallberg $\mathrm{M}$, Wang $\mathrm{H}$, Wang $\mathrm{S}$, Peng J, Wang $\mathrm{Z}$, Lu H, et al. Template-based protein structure modeling using the RaptorX web server. Nat Protoc. 2012;7(8):1511-22. 
44. Warde-Farley D, Donaldson SL, Comes O, Zuberi K, Badrawi R, Chao P, et al. The GeneMANIA prediction server: biological network integration for gene prioritization and predicting gene function. Nucleic Acids Res. 2010;38(Web Server issue):W214-20.

45. Kochumon SP, Yesodharan D, Vinayan K, Radhakrishnan N, Sheth JJ, Nampoothiri S. GM2 activator protein deficiency, mimic of Tay-Sachs disease. International Journal of Epilepsy.

2017;4(02):184-7. 